UCB-PTH-08/65

\title{
Neutrino Masses, Baryon Asymmetry, Dark Matter and the Moduli Problem - A Complete Framework
}

\author{
Piyush Kumar \\ Department of Physics, University of California, \\ Berkeley, CA 94720 USA \\ and \\ Theoretical Physics Group, \\ Lawrence Berkeley National Laboratory, \\ Berkeley, CA 94720 USA
}

Recent developments in string theory have led to "realistic" string compactifications which lead to moduli stabilization while generating a hierarchy between the Electroweak and Planck scales at the same time. However, this seems to suggest a rethink of our standard notions of cosmological evolution after the end of inflation and before the beginning of BBN. This epoch is crucial for addressing the issues of neutrino masses, baryon asymmetry, Dark Matter (DM) abundance and the moduli (gravitino) problem. We argue that within classes of realistic string compactifications as defined above, there generically exists a light modulus with a mass comparable to that of the gravitino which is typically much smaller than the Hubble parameter during inflation. Therefore, it is destabilized and generates a large latetime entropy when it decays. Thus, all known elegant mechanisms of generating the baryon asymmetry of the Universe in the literature have to take this fact into account.

In this work, we find that it is still possible to naturally generate the observed baryon asymmetry of the Universe as well as light left-handed neutrino masses from a period of Affleck-Dine (AD) leptogenesis shortly after the end of inflation, in classes of realistic string constructions with a minimal extension of the MSSM below the unification scale (consisting only of right-handed neutrinos) and satisfying certain microscopic criteria described in the text. The AD mechanism has already been used to generate the baryon asymmetry in the literature; however in this work we have embedded the above mechanism within a framework well motivated from string theory and have tried to describe the epoch from the end of inflation to the beginning of BBN in a complete and self-consistent manner. The consequences of our analysis are as follows. The lightest left-handed neutrino is required to be virtually massless. The moduli (gravitino) problem can be naturally solved in this framework both within gravity and gauge mediation. The observed upper bound on the relic abundance constrains the moduli-matter and moduli-gravitino couplings since the DM is produced nonthermally within this framework. Finally, although not a definite prediction, the framework naturally allows a light right-handed neutrino and sneutrinos around the electroweak scale which could have important implications for the nature of DM as well as the LHC. 
Contents

I. Introduction

II. Framework of "Realistic" String Compactifications

III. Cosmology within the Framework

A. Evolution during the Inflationary Phase

B. Post-Inflationary Evolution

1. Generation of Lepton Number

2. Satisfying All Constraints

C. The Baryon Asymmetry

D. Moduli (Gravitino) Problem and Non-thermal Dark Matter

IV. Microscopics

A. $U(1)_{s}$, Anomalies, Gauge Invariance and Brane Instantons

B. Application to Neutrinos

C. Microscopic Constraints to obtain a small $\frac{h^{2}}{\lambda}$

2

1. Majorana Operator Domination (see-saw case)

2. Weinberg Operator Domination

V. Other String/ $M$ Theory Compactifications

VI. Potential Consequences for Observable Physics

VII. Conclusions

Acknowledgments

\section{A. Lightest "Modulus" in Gauge Mediation}

B. What if $\hat{\tilde{N}}_{3}$ is displaced during inflation?

C. Technical Details for computing the Lepton Number

D. D-term contribution to masses of Moduli

References

\section{INTRODUCTION}

Many theoretical and observational advances have been made to uncover the mysteries of the very early Universe. Recent cosmological observations from WMAP seem to favor an inflationary phase of the 
Universe $^{1}$. Although, there still does not exist an agreed-upon microscopic theory of inflation, there has been a lot of progress in this direction in recent years. The theory of the primordial synthesis of nuclei $\mathrm{BBN}$, which starts at a temperature of about an $\mathrm{MeV}$, is also quite successful in explaining observations. The evolution of the Universe after BBN - leading to decoupling of matter and radiation, traditional matter domination and large-scale structure formation, is also fairly well understood.

However, not much is known about the epoch from the end of inflation to the beginning of BBN. Theoretically, physics during this epoch has been less studied as a whole (relatively speaking) compared to the inflationary epoch. However, this epoch is quite important for a number of reasons. For example, a crucial property of the Universe, the existence of a baryon asymmetry, has to be explained during this epoch, viz after the end of inflation and before BBN. This is because, inflation, in addition to successfully diluting dangerous relics from the early past such as monopoles, domain walls, cosmic strings, etc., also dilutes any pre-existing baryon asymmetry. Therefore, the baryon asymmetry has to be generated after the inflationary epoch. The existence of baryon asymmetry requires that the Sakharov criteria be satisfied. The three most popular ways of satisfying these by a) the Affleck-Dine mechanism, b) out-of-equilibrium decay of a heavy particle (as in GUT baryogenesis, thermal leptogenesis, resonant soft leptogenesis) and c) during the electroweak phase transition (as in electroweak baryogenesis) all happen before BBN. Various particle-physics models of baryogenesis in this epoch incorporating the above mechanisms, especially thermal leptogenesis [1], resonant soft leptogenesis [2], affleck-dine baryogenesis [3] and leptogenesis [4] and electroweak baryogenesis [5] have been considered in the literature. The origin of neutrino masses can be linked to the generation of baryon asymmetry in models of leptogenesis, providing an opportunity to solve both outstanding problems at the same time. In this sense, the framework of leptogenesis is quite appealing.

Many beyond-the-Standard-Model (BSM) particle physics models well motivated from a microscopic theory such as string theory, also have additional scalar particles known as "moduli". These moduli are scalar fields which couple very weakly to the visible sector and scale like ordinary matter. Thus, they typically dominate the energy density of the Universe and could decay after BBN spoiling its successes, and at the same time greatly diluting any previously generated baryon asymmetry. Therefore, this provides a serious constraint to all existing mechanisms for producing the baryon asymmetry. The "cosmological moduli problem" is therefore quite undesirable. In supersymmetric extensions of the Standard-Model, the overproduction of gravitinos can cause similar problems. In addition, the "standard" picture in which the Universe is radiation dominated during the whole epoch can be significantly altered in the presence of moduli. In particular, Dark Matter (DM) particles, instead of being produced during a phase of thermal equilibrium, are typically dominantly produced non-thermally, via the direct decay of moduli. However, this can lead to further problems since it is easy to produce too much dark matter compared with what we observe today. To summarize, therefore, understanding the epoch starting from the end of inflation to the beginning of $\mathrm{BBN}$ is extremely crucial to addressing all the above issues in a systematic and holistic manner.

These problems have been known for a long time, various aspects of which have been discussed in the literature in the context of supergravity and string theory [6]. However, early investigations of these issues, although important, were not very concrete as moduli stabilization in string compactifications and

\footnotetext{
${ }^{1}$ In this work, we will assume that the inflationary paradigm is correct.
} 
the resulting spectra of moduli was not well understood. With great improvement in our understanding of moduli stabilization in recent years, these issues warrant a careful investigation in light of the new developments.

The aim of this work is to do precisely that - address the above issues in a systematic manner within a well-motivated and complete framework - that provided from string theory. To be clear, the goal is not to construct an explicit model arising from a particular string construction. Instead, the goal is to figure out the microscopic conditions required to address and solve the above cosmological issues by utilizing only generic features of classes of well-motivated low energy effective-field-theories (EFTs) arising in various string theory compactifications. More precisely, by "well-motivated" it is meant that the string compactifications stabilize the moduli as well as generate a stable hierarchy between the electroweak and planck scales. With developments in string compactifications in recent years, it is now possible to realize these features in a natural manner. In particular, in this paper we will work within the framework of low energy supersymmetry.

The results obtained from such an analysis are quite encouraging and can be summarized simply as follows. Within classes of string compactifications with a mechanism of generating a stable hierarchy and stabilizing the moduli at the same time, there is generically a light modulus (moduli) whose mass is comparable to the gravitino mass scale and generically much smaller than the Hubble parameter during inflation $\left(H_{\text {inf }}\right)$. The modulus is therefore typically displaced from its minimum, dominates the energy density of the Universe for a long time and its decay (close to BBN) generates a large entropy which greatly dilutes any pre-existing baryon asymmetry. Therefore, existing mechanisms of baryogenesis have to take this feature into account. We find, in particular, that the mechanism of Affleck-Dine leptogenesis (via the $L H_{u}$ flat-direction) can still generate the observed baryon asymmetry as well as light neutrino masses of the Universe shortly after the end of inflation in classes of string constructions with a minimal extension of the MSSM below the unification scale - just consisting of right-handed neutrinos, provided certain microscopic criteria are satisfied. These are as follows. The spectra should consist of a gauged $U(1)_{B-L}$ symmetry with right handed neutrinos in addition to that of the MSSM (and possible vector-like exotics). The gauged $U(1)_{B-L}$ symmetry must be such that the $U(1)_{B-L}$ gauge boson gets a Stuckelberg mass. String instantons with the appropriate zero-mode structure must be present in order to give rise to the appropriate Majorana or Weinberg operators generating neutrino masses. Because majorana masses are generated by instantons and are exponentially suppressed relative to the $B-L$ breaking scale, it is natural for the right-handed neutrinos to be hierarchical and the heaviest right-handed neutrino to not be displaced from its minimum during inflation, which is crucial for generating a non-zero baryon number. Furthermore, generating the correct amount of baryon asymmetry requires that the neutrino yukawa couplings be very small and that the lightest left-handed neutrino is virtually massless ${ }^{2}$. In an ordinary effective theory, a very small yukawa coupling and lightest neutrino mass may not look very natural; however since the yukawa couplings in the microscopic constructions we are interested in are exponentially suppressed, it is natural for them to be small. The mechanism of generating the baryon asymmetry is similar to the ones described in [7, 8] where one also requires a very light lightest neutrino (although not as small as required here). However, in this work we have embedded the mechanism (with subtle differences in details) in a complete framework well-motivated from string theory.

${ }^{2}$ It has an extremely small mass $\sim 10^{-16} \mathrm{eV}$. 
The above framework can also naturally solve the moduli (gravitino) problem in both gravity mediation and gauge mediation, although in a different manner. Within gravity mediation, there is no moduli (gravitino) problem if the lightest modulus is of $\mathcal{O}\left(m_{3 / 2}\right) \gtrsim 10 \mathrm{TeV}^{3}$. Within gauge mediation, the lightest "modulus" (scalar field) is typically again of $\mathcal{O}\left(m_{3 / 2}\right)^{4}$ although $m_{3 / 2}$ is much smaller $(\leq \mathrm{GeV})$ and stable. So, the main constraint in this case comes from the decay of this modulus to the gravitino. The dark matter (DM) candidate, be it the LSP or the gravitino, is dominantly produced non-thermally within this framework. Therefore, the observed upper bound on the relic density provides an important constraint on the moduli-matter and the moduli-gravitino couplings arising within this framework. Finally, although not a definite prediction of the above framework, the framework allows an interesting possibility at the $\mathrm{LHC}$ - that of a right-handed neutrino at the $\mathrm{TeV}$ scale, but without the presence of an additional $U(1)$ gauge boson at that scale. This could give rise to interesting predictions for the LHC and Dark Matter (DM) and could in principle be distinguished from scenarios with $\mathrm{TeV}$ scale right-handed neutrinos in which an additional $U(1)$ gauge boson at around the same scale is also present.

To our knowledge, this is the first time that a well defined microscopic framework has been outlined to address all cosmological issues starting from the end of inflation to the beginning of BBN in a manner so as to be consistent with low energy supersymmetry and giving rise to interesting physics at the LHC. Each of the above microscopic conditions are naturally compatible with each other and have been shown to be true separately within large classes of constructions. Therefore, we expect that there should exist a reasonably large class of constructions within the sub-landscape of "realistic" string theory vacua in which all of them are satisfied simultaneously, leading to a successful solution of all the above cosmological issues in a natural manner.

The outline of the paper is as follows. Section $\amalg$ explains the framework of string theory compactifications which are well-motivated from a microscopic viewpoint and also have many desirable features from the standpoint of low-energy physics. The features of the framework concerning the moduli and matter spectra which are most relevant for addressing the above mentioned cosmological issues are described in some detail. Section III is a detailed discussion of cosmology within the framework, in particular the mechanisms which generate the baryon asymmetry in the presence of moduli and also addresses the moduli problem and generation of adequate amount of Dark Matter. The details of the microscopic structure relevant for the framework, as well as the constraints on the microscopic parameters to get the correct baryon asymmetry, are discussed in section IV. Section V briefly discusses the realization of the framework studied in other corners of string/ $M$ theory, in particular the low energy limit of $M$ theory compactifications. In section VI, some broad potential consequences for physical observables are outlined. We conclude in section VII and discuss future directions. In appendix $\mathrm{A}$, the mass scale of the lightest modulus is estimated within gauge mediation. In appendix $\mathrm{B}$, it is shown that it is not possible to generate a baryon asymmetry by affleck-dine leptogenesis if the heaviest right-handed sneutrino is displaced from its minimum after inflation. Appendix $\mathbb{C}$ deals with some technical details concerning the computation of the lepton number shortly after inflation, and appendix $\mathrm{D}$ estimates the $D$-term contribution to the masses of the kähler moduli appearing in the various $D$-terms.

\footnotetext{
${ }^{3}$ It is still possible to obtain $\mathcal{O}(100) \mathrm{GeV}$ superpartner spectra within both gravity and gauge mediation so as to be interesting at the LHC.

4 within a few orders of magnitude.
} 


\section{FRAMEWORK OF "REALISTIC"STRING COMPACTIFICATIONS}

In this section, we give a brief and not very technical review of relevant aspects of string compactifications giving rise to vacua with many desirable low energy features. This will be helpful for setting the stage in which all the above cosmological issues can be addressed systematically. In particular, we will be interested in the spectra of moduli in realistic string compactifications. This will be crucial for the cosmological evolution of the Universe after inflation. The reader primarily interested in cosmology may skip this section, nevertheless it will be useful to remember that in realistic string compactifications with low energy supersymmetry, there generically exists a light modulus with mass comparable to $m_{3 / 2}$, which is typically much smaller than the Hubble parameter during inflation $\left(H_{\text {inf }}\right)$.

The most important challenges to constructing a low energy theory arising from a string compactification are related to dynamical issues such as moduli stabilization, supersymmetry breaking and explaining the Hierarchy between the Electroweak and Planck scales. Successfully addressing these opens the possibility to construct models of particle physics beyond the Standard-Model (SM) within the framework of string theory and study them to the extent that testable predictions for real observables in particle physics and cosmology can be made. However, in carrying out this program in string theory, it has to be kept in mind that the properties of beyond-the-Standard-Model (BSM) particle physics models are intimately connected to the dynamical issues mentioned above. This is because the masses and couplings of the particle physics models depend on the properties of the vacuum (or class of vacua) of the underlying string theory compactifications, in particular, the values of the moduli in the vacuum. In recent years, substantial progress has been made in the past few years towards addressing the above dynamical issues within various corners of the entire $M$ theory landscape, see [9, 10, 11, 12]. For definiteness, we will consider Type IIB string compactifications on Calabi-Yau orientifolds where the results are best understood. However, the arguments given below are quite general and only depend on certain qualitative features. Hence, these could be generalized to many other known classes of compactifications.

\section{A. Moduli Spectra and Low-energy Supersymmetry}

We are interested in estimating the spectra of moduli masses in "realistic" string compactifications where both the moduli and the Hierarchy are stabilized at the same time. For concreteness, we will focus on string compactifications with low energy supersymmetry. Regarding the unification of gauge couplings within the MSSM at $M_{G U T} \sim 10^{16} \mathrm{GeV}$ as an important clue, in our analysis we restrict to string compactifications with a high compactification and string scale $\left(M_{G U T} \approx M_{K K} \lesssim M_{s} \sim 10^{17} \mathrm{GeV}\right)^{5}$. Another reason for such a restriction is that $M_{s} \ll M_{G U T}$, or equivalently, a very large compactification volume $\mathcal{V}$, gives rise to significantly more serious problems with BBN as will be seen later.

By low-energy supersymmetry it is meant that the scale of superpartners has to be of $\mathcal{O}(\mathrm{TeV})$ in order to stabilize the higgs mass. String compactification frameworks with moduli stabilization and supersymmetry breaking leading to low energy supersymmetry are of the following type: supersymmetry is broken in some hidden sector by a combination of matter and moduli fields and mediated to the visible

\footnotetext{
${ }^{5}$ The discrepancy of an order of magnitude between $M_{s}$ and $M_{K K}$ can be naturally explained by threshold corrections, so we will take all of them to be roughly of the same scale.
} 
sector predominantly by exchange of closed strings (gravity mediation) or open strings (gauge mediation). What dominates depends on the separation $(d)$ between the hidden sector (which breaks supersymmetry) and the visible sector, compared to the string length $\left(l_{s}\right)$. In cases in which moduli stabilization is best understood, $d \gg l_{s}$ which gives rise to gravity mediation. Therefore, we will discuss gravity mediation in more detail, although we will also comment on gauge mediation models within string theory later.

Intuitively, the above claim can be understood as follows. In models with a vanishing (tiny) cosmological constant, the gravitino mass in $\mathcal{N}=1$ supergravity can be written as:

$$
m_{3 / 2}=e^{K / 2} \frac{W}{m_{p}^{2}} \approx \frac{\sqrt{\sum_{i} F^{i} F_{i}}}{m_{p}}
$$

where $F^{i}$ correspond to fields which have non-zero $F$-term vevs. In order to explain the Hierarchy, the gravitino mass $m_{3 / 2}$ has to vastly suppressed relative to $m_{p}$, implying that $F_{i}$ has to be suppressed relative to $m_{p}^{2}$. This implies from above that $W$ (which depends on moduli) must also be suppressed ${ }^{6}$ relative to $m_{p}^{3}$. A natural way to obtain a small $W$ is by an exponential suppression ${ }^{7}$. In a generic situation, the curvature of the scalar potential at the minimum which determines the mass of the modulus appearing in the exponential, is of $\mathcal{O}\left(\frac{W^{2}}{m_{p}^{4}}\right)$, or equivalently of $\mathcal{O}\left(m_{3 / 2}^{2}\right)$ from (1). The precise value, however, depends on details. We will carry out a detailed version of this simple argument within Type IIB compactifications. For completeness, we will estimate the masses of other moduli as well.

Any string compactification preserving $\mathcal{N}=1 \mathrm{SUSY}$ in four dimensions can be written at low energies in terms of $\mathcal{N}=1, D=4$ SUGRA, which at the two-derivative level is completely specified by a Kähler potential, superpotential and gauge kinetic function. For IIB compactifications, these are given by:

$$
\begin{aligned}
K & =-2 \log \left(\mathcal{V}\left(T_{i}, V_{i}\right)\right)-\log \left(i \int \Omega \wedge \Omega\left(U_{i}\right)\right)-\log (S+\bar{S})-\hat{K}\left(Y_{i}+\bar{Y}_{i}\right)+\tilde{K}_{\alpha \beta} \bar{Q}_{\alpha} Q_{\beta}+\ldots \\
W & =W_{f l u x}+W_{n p}+W_{\text {matter }} \\
& =m_{p}^{3}\left(\frac{1}{\alpha^{\prime}} \int G_{3} \wedge \Omega\left(S, U_{i}\right)+\sum_{i} A_{i}\left(U_{j}, V_{k}\right) e^{-a_{i}\left(T_{i}+h_{i}(F) S\right)}\right)+\lambda e^{-S\left(T_{m}\right)} Q_{\alpha} Q_{\beta}+y_{\alpha \beta \gamma}\left(U_{j}, V_{k}\right) Q_{\alpha} Q_{\beta} Q_{\gamma}+\ldots \\
f_{a} & =T_{a}+h_{a}(F) S
\end{aligned}
$$

The kähler potential gets contributions from the moduli and matter fields. The contribution of matter fields can be expressed as an expansion around the origin as seen from $(2)^{8}$. $\mathcal{V}$ denotes the volume of the internal manifold in units of the string length $\left(l_{s}\right)$ and depends on the kähler $\left(T_{i}\right)$ and open string moduli $\left(V_{i}\right)$. $\Omega$ corresponds to the unique holomorphic three-form of the Calabi-Yau manifold and depends on the complex structure moduli $\left(U_{i}\right)$ while $G_{3}$ corresponds to a three-form field strength present in Type IIB string theory which depends on the dilaton $(S) . \tilde{K}_{\bar{\alpha} \beta}$ is the kähler metric of the visible matter fields $Q_{\alpha}$. The superpotential, in addition to the classical flux contribution which depends on $S$ and $U_{i}$, and the non-perturbative contribution which depends on the kähler moduli $\left(T_{i}\right)$ and the dilaton, also has a matter contribution. The renormalizable matter superpotential contains yukawa couplings which depend

\footnotetext{
${ }^{6}$ assuming that $e^{K / 2}$ does not give a huge suppression, which is true for compactifications with a large string scale $M_{s} \gtrsim$ $M_{G U T}$.

7 there could be a small constant piece in addition as well.

8 This is because most matter fields are supposed to have vanishing vevs.
} 
on the complex structure and open string moduli and also contains potential mass terms which depend on the kähler moduli, as can be seen from (2). In addition, the superpotential and the kähler potential could also have non-renormalizable terms; these have been suppressed above. The potential mass terms will be crucial for the generation of majorana neutrino masses as we will see later. Finally, the gauge kinetic function depends primarily on the kähler moduli; however demanding a chiral matter sector on the world-volume of the gauge theory implies that there is also a dependence on the dilaton which depends on certain topological data $h_{a}(F)$.

The fluxes generate contributions to the energy density of the order of the string scale $M_{s} \equiv \frac{1}{\sqrt{\alpha^{\prime}}}$, which is the natural scale in the problem. Taking proper account of the weyl rescaling to Einstein frame, the masses of the moduli stabilized by bulk and brane worldvolume fluxes (the dilaton, complex structure and open string moduli) can be estimated as [13]:

$$
m_{S, U_{i}, V_{i}} \sim \frac{\alpha^{\prime}}{R^{3}} \equiv \frac{M_{K K}^{3}}{M_{s}^{2}}
$$

Here, $M_{K K}^{-1} \equiv R$ is the typical size of the bulk of the Calabi-Yau. These moduli are stabilized supersymmetrically, so they have negligible $F$-term components. Since these moduli have masses of $\mathcal{O}\left(10^{16} \mathrm{GeV}\right)$, below these energies one could integrate them out and obtain an effective constant flux superpotential $W_{0}$.

The Kähler moduli are not stabilized by fluxes. However, non-perturbative effects can in general give rise to a dependence on these moduli and hence help in stabilizing them. It has been shown that some Kähler moduli $\left(T_{i}\right)$ can be stabilized dominantly by non-perturbative effects. However, because of the chirality of the MSSM (and possible extensions), at least the Kähler modulus which measures the volume of the cycle on which the SM gauge group is supported cannot be stabilized purely by non-perturbative effects [14]. This is also true for the modulus $T_{m}$ appearing in the superpotential in (2). Therefore, some Kähler moduli $\left(T_{\alpha}\right)$ have to be stabilized by a combination of other effects (arising from $D$-terms [15], Kähler corrections [16], moduli trapping [17], etc.) and non-perturbative effects. As shown in [18], the moduli $T_{i}$ are stabilized supersymmetrically at leading order while the remaining ones $T_{\alpha}$ are not. This generically leads to masses for $T_{i}$ which are parametrically larger than $m_{3 / 2}$ by a factor $\sim a_{i}\left\langle T_{i}\right\rangle$. The masses of moduli which are stabilized primarily by contributions from the Kähler potential are of the same order as $m_{3 / 2}{ }^{9}$, while $D$-term contributions to masses of moduli could be much larger than $m_{3 / 2}$, as estimated in appendix D,

The non-perturbative effects in the superpotential $\left(W_{n p}\right)$ should be of the same order as $W_{0}$ to obtain a minimum if the volume $\mathcal{V}$ is not too large ${ }^{10}$. Thus the flux superpotential $W_{0}$ has to be suppressed, just like $W_{n p}$. This can be naturally provided by the discrete tuning of fluxes. In fact, it is also possible to choose fluxes such that $W_{0}$ vanishes [15]. In the presence of warping, there are also throat moduli $\left(Y_{i}\right)$ which are stabilized by the fluxes, their vacuum values related to the warp factor $\left(e^{A_{\text {min }}}\right)$ at the tip of the throat. In particular [13]:

$$
m_{Y_{i}} \sim e^{A_{\min }} \frac{\alpha^{\prime}}{R^{3}}=e^{A_{\min }} \frac{M_{K K}^{3}}{M_{s}^{2}}
$$

\footnotetext{
${ }^{9}$ If $\mathcal{V}$ is not too large, then the mass of the overall modulus is also of the same order as $m_{3 / 2}$.

10 This is true in particular if $M_{G U T} \approx M_{K K} \lesssim M_{s}$ as has been assumed.
} 
Without additional effects, the vacuum obtained after stabilizing the moduli generically has negative vacuum energy, i.e. it is an anti de-Sitter (AdS) vacuum. Therefore, a positive contribution to the vacuum energy is needed to obtain a $\mathrm{dS}$ vacuum with a positive cosmological constant. Moreover, the positive contribution has to be (finely) tuned so that the cosmological constant has the observed value, for which no satisfactory dynamical solution exists at present. Various mechanisms giving rise to a positive contribution to the vacuum energy exist, such as explicit supersymmetry breaking contributions from anti D-branes, or from $F$-term and $D$-term uplifting by matter fields. Since our primary interest is the spectra of moduli (scalar fields), the masses of new scalar degrees of freedom which appear in the mechanisms above must also be taken into account after fine-tuning the cosmological constant. $F$-term uplifting, for example, generically gives rise to masses for these matter fields of $\mathcal{O}\left(m_{3 / 2}\right)[12$, 19], while $D$-terms could give rise to large masses for the moduli, as estimated in appendix D.

We would also like to comment on gauge mediation models within string theory. One could try to imagine a situation in which all moduli are stabilized at a high scale in an almost supersymmetric and minkowski vacuum. Although there do not currently exist explicit compactifications which realize this situation (however, see [20] for some work in this direction), but one could hope that future developments could accomplish this. Supersymmetry could then be broken by a dynamical mechanism in a hidden matter sector and be mediated to the visible sector by gauge interactions if $d \leq l_{s}$. This is the philosophy of many local models in string theory [21]. However, it is important to note that even within such gauge mediation models, there exists a modulus-like scalar field (the scalar partner of the goldstino, or the $D$-flat direction comprising the vector-like messengers) which generically gets a mass comparable to $m_{3 / 2}$ (within a few orders of magnitude). This is argued in appendix A for generic models of gauge mediation.

It is important to note that in compactifications in which the volume $\mathcal{V}$ is very large (or equivalently, $M_{s}$ is much smaller than the traditional $M_{G U T}$ ), such as which could arise in LARGE volume compactifi-

cations [22] or in the local models above if they are sufficiently decoupled from gravity, the overall volume modulus could be much lighter than $m_{3 / 2}$. Thus, these models would cause very serious problems for BBN unless mechanisms exist which could sufficiently dilute the entropy produced at late times by the decay of these extremely light moduli. Although this is possible in principle, there do not exist concrete mechanisms within string theory at present which realize it. Therefore, as mentioned earlier, we do not consider this situation.

To summarize, realistic string compactifications with a mechanism of generating and stabilizing the Hierarchy between the electroweak and Planck scales while stabilizing the moduli, give rise to two sets of moduli - one very heavy and one light. The light moduli are typically comparable to $m_{3 / 2}$. With low energy supersymmetry, the hubble parameter during inflation $H_{\text {inf }}$ is typically much larger than the moduli or gravitino mass, which generically destabilizes the light moduli. This feature is expected to be true for other classes of string compactifications as well. For example, the above feature is satisfied for moduli spectra in realistic $M$ theory compactifications studied in [23]. Hence, we will assume the above spectra of moduli henceforth.

\section{B. Visible Sector Model Building}

Now that we understand some of the important features of the class of vacua obtained in the above framework, the next step is to look at fluctuations around these vacua, those pertaining to matter and 
gauge degrees of freedom. This corresponds to constructing the matter and gauge spectrum comprising a beyond-the-SM particle physics model. As explained earlier, this is not the subject of this paper. Nevertheless, it is worthwhile to elaborate a little on visible sector model-building.

Most work on explicit string model-building is focussed on computing spectra on compactifications on toroidal orbifolds/orientifolds and Gepner models, where CFT techniques are available. Computing spectra on a general compact Calabi-Yau is extremely challenging. However, considerable progress has been made in computing spectra in non-compact (local) Type II constructions with D-branes at singularities where gravity can be decoupled at leading order. In both cases, semi-realistic spectra for beyond-the-SM physics have been constructed. We will not concern ourselves with constructing specific singularities or toroidal constructions realizing the MSSM or its extension thereof; rather we are interested in studying generic features which are crucial in solving the cosmological problems outlined in the introduction and would be relevant even if a particular explicit construction is not.

Leptogenesis provides us with an elegant mechanism of explaining the origin of neutrino masses as well as the baryon asymmetry of the Universe in one theoretical framework. Therefore, with the principle of Occam's Razor in mind, in this work we will focus on leptogenesis as providing the mechanism for baryon asymmetry of the Universe. The requirement of small neutrino masses requires the presence of the lepton number violating operator $-\frac{\kappa}{M} L H_{u} L H_{u}$ in the superpotential, with $\kappa$ a dimensionless coupling and $M$ a large mass-scale. There are two ways of generating this operator - a) It may be present in the microscopic construction itself ("Weinberg" case) and/or b) It may be generated at lower energies by integrating out right-handed neutrinos $\left(N_{R}\right)$ which appear in many semi-realistic spectra arising from string constructions ("see-saw case"). These constructions typically have an additional gauged $U(1)-U(1)_{B-L}$ to be precise. The existence of a gauged $U(1)_{B-L}$ is quite natural as it is the unique flavor independent anomaly-free $U(1)$ asymmetry, and string theory only allows gauge symmetries. Anomaly cancellation (including the gravitational anomaly) in fact requires the existence of three families of right-handed neutrinos. However, for this mechanism to work, large majorana masses for right handed neutrinos have to be generated. Both of the above mechanisms will be discussed within the context of string constructions in section IVB.

For concreteness, in the following, we will assume that the visible sector consists of a supersymmetric standard model with a $U(1)_{B-L}$ gauge group in addition to that of the SM and a matter spectrum consisting of the MSSM, possibly vector-like exotics and three right-handed neutrinos.

\section{COSMOLOGY WITHIN THE FRAMEWORK}

In this section, we will discuss the cosmological implications of the framework described above during and after inflation until the beginning of BBN. As stated earlier, we will assume the existence of an inflationary phase in the very early Universe which solves the flatness and horizon problems and also gives rise to almost scale invariant density perturbations as observed.

Many supersymmetric models such as the MSSM commonly have a degenerate set of vacua at the level of renormalizable terms, meaning that there are many directions in the space of scalar fields where the potential vanishes classically in the supersymmetric limit with $m_{p} \rightarrow \infty$. These directions are therefore known as "flat-directions". These flat-directions are, however, lifted by supersymmetry breaking and non-renormalizable terms in the superpotential [24]. We will specifically be interested in the evolution of moduli, sneutrinos and "flat-directions" during and after inflation as it will be crucial for the estimation 
of the baryon asymmetry.

\section{A. Evolution during the Inflationary Phase}

As explained in [24], the finite energy density of the Universe during inflation breaks supersymmetry. The finite energy supersymmetry breaking is transmitted to the moduli, sneutrinos and flat-directions by the cross-coupling of the inflaton (which dominates the energy density during inflation by definition) and the above fields. Ordinary hidden sector supersymmetry breaking corrections to the scalar potential are much smaller during inflation since $H_{I} \gg m_{3 / 2}$ in vacua with low energy supersymmetry. Parameterizing all these couplings, one finds the following contribution to the scalar potential for the moduli, sneutrinos (if present in the spectrum) and flat directions $(\phi)$ during inflation [24]:

$$
\begin{aligned}
V_{\text {inf }}= & \sum_{i=1}^{N} c_{1}^{i} H_{I}^{2}\left|X_{i}\right|^{2}+M_{X_{i}}^{2}\left|X_{i}\right|^{2}+\left(b_{1}^{i} H_{I} M_{X_{i}} X_{i} X_{i}+c . c\right)+\ldots \\
& +c_{2}^{k} H_{I}^{2}\left|\tilde{N}_{k}\right|^{2}+M_{N}^{k}\left|\tilde{N}_{k}\right|^{2}+\left(b_{2} H_{I} M_{N}^{k} \tilde{N}_{k} \tilde{N}_{k}+c . c\right)+. . \\
& +c_{3} H_{I}^{2}|\phi|^{2}+\left(\frac{a \lambda H_{I} \phi^{n}}{n M^{n-3}}+\text { c.c. }\right)+|\lambda|^{2} \frac{|\phi|^{2 n-2}}{M^{2 n-6}}+\left(m_{0}^{2}|\phi|^{2}+\frac{A m_{3 / 2} \lambda \phi^{n}}{n M^{n-3}}+c . c\right)+\ldots
\end{aligned}
$$

Here $\left\{c_{1}^{i}, c_{2}^{k}, c_{3}\right\},\left\{b_{1}^{i}, b_{2}\right\}$ and $\{a, A, \lambda\}$ are model-dependent coefficients typically of $O(1)$ but can be of either sign. From (5), we see that the moduli $X_{i}$ and sneutrinos $\tilde{N}$ have a mass term in the potential because the moduli are stabilized and the sneutrinos have a supersymmetric majorana mass term. The flat directions $\phi$ on the other hand are massless at leading order by definition. The scalar potential depends on $\phi$ only through supersymmetry breaking (both hubble induced and hidden sector) and by nonrenormalizable terms in the superpotential. These terms are also present for the moduli and sneutrinos. However, the hidden sector supersymmetry breaking and non-renormalizable terms are not written for simplicity as they are much smaller.

We will start by studying the evolution of moduli during inflation. In most natural models of inflation, the hubble parameter during inflation $\left(H_{I}\right)$ is $\mathcal{O}\left(10^{13}-10^{14}\right) \mathrm{GeV}$. For our purposes, we will assume $H_{I} \sim 10^{12}-10^{13} \mathrm{GeV}$ for our solutions to be consistent conservatively, as will be seen later. For larger values of $H_{I}$, it is still possible for our solutions to be consistent but less parameter space is available. As stated earlier, the coefficients $c_{1}^{i}$ depend on the concrete model of inflation and can be of either sign. If they turn out to be positive and $\mathrm{O}(1)$, then from (5) it is clear that the effective mass-squared parameter for all the moduli $\left(M_{X_{i}}^{e f f}\right)^{2}=M_{X_{i}}^{2}+c_{1}^{i} H_{I}^{2}$ is positive and $\gtrsim \mathcal{O}\left(H_{I}^{2}\right)$. However, a negative sign for $c_{1}^{i}$ is possible for non-minimal couplings between the inflaton and the relevant field, which is in fact a generic possibility within string theory. In this case, the effective mass-squared parameter for the moduli are given by:

$$
\left(M_{X_{i}}^{e f f}\right)^{2}=M_{X_{i}}^{2}-\left|c_{1}^{i}\right| H_{I}^{2}
$$

As explained in section 【IA the complex structure $\left(U_{i}\right)$, dilaton $(S)$ and open string moduli $\left(V_{i}\right)$ naturally obtain masses of $\mathcal{O}\left(10^{16}\right) \mathrm{GeV}$. Therefore, the effective mass-squared parameter for these moduli $\left(M_{X_{\text {heavy }}}^{\text {eff }}\right)^{2}$ is still positive and they settle down to the true (late time) minima in about a hubble time. They are thus not displaced from their true minima during inflation. The masses of the throat moduli 
$\left(Y_{i}\right)$ are $\mathcal{O}\left(10^{11}-10^{12}\right) \mathrm{GeV}$. So their fate depends more on the concrete model of inflation and the magnitude of the $c_{1}^{Y_{i}}$ in particular. For negative $c_{1}^{i}$ slightly small in magnitude for some reason $(\lesssim 0.1)$, the moduli $Y_{i}$ will not be displaced, otherwise they will. Finally moving on to the light moduli, which we denote by $X_{\text {light }}$ in general, we see from section IIA that they have masses of $\mathcal{O}\left(m_{3 / 2}\right)$, i.e. of $\gtrsim 10$ $\mathrm{TeV}^{11}$. Therefore, these moduli will generically be displaced from their true minima for a wide range of $c_{1}^{i}$. As will be shown later, our final conclusions regarding the baryon asymmetry and dark matter will not change whether or not the moduli $Y_{i}$ are displaced, as long as the light moduli $X_{\text {light }}$ are displaced during inflation. Therefore, for simplicity and concreteness, it will be assumed that all the heavy moduli $\left(m_{X_{\text {heavy }}} \gg m_{3 / 2}\right)$ settle in their true minima and the light moduli $X_{\text {light }}$ are displaced from their true minima by a large amount.

The situation with sneutrinos is quite interesting. As explained in section IIB, many BSM models for new physics arising from string constructions have a $U(1)_{B-L}$ symmetry with three families of righthanded neutrinos (and sneutrinos in a supersymmetric model). In such constructions, for the right handed neutrinos and sneutrinos to receive a majorana mass term, the $U(1)_{B-L}$ symmetry needs to be broken. However, until recently it has proven difficult to break the above symmetry in the desired manner without not producing other undesirable effects such as dangerous $B$ and $L$ violating operators at the same time. It was recently shown in [25] that under certain conditions, the perturbatively forbidden majorana mass term could be generated in a natural way by stringy non-perturbative effects (see section IV for details). These non-perturbative effects arise from euclidean brane instantons; hence the majorana masses generated from these effects are exponentially suppressed. The scale at which the gauged $U(1)_{B-L}$ is broken is roughly the string scale, giving rise to a mass for the $B-L$ gauge boson of $\mathcal{O}\left(M_{s}\right)$. This gives rise to a mass term in the superpotential for the right-handed neutrinos (the third term in the expression for the superpotential in (2)). The majorana masses are given by:

$$
M_{N}^{a b} \equiv \lambda v_{B-L}=e^{K / 2} \sum_{r} d_{a}^{r} d_{b}^{r} e^{-S_{E 3}^{(M), r}\left(T_{m}\right)} m_{p}
$$

where $\lambda$ is a dimensionless coefficient, $d_{a}^{r}, d_{b}^{r}$ are constants generically of $\mathcal{O}(1)$, and $S_{E 3}^{(M), r}$, the action of the $r^{\text {th }}$ instanton, is (classically) equal to the world-volume of the instanton. For details, refer to section IVB, To generate majorana masses for three right-handed neutrinos, three or more contributing instantons are required. Since the world-volume of each instanton will in general differ by $\mathcal{O}(1)$, the flavor structure above implies that there will generically be a hierarchy among the right handed majorana mass eigenvalues. This implies that it is very natural for the heaviest right-handed sneutrino $\tilde{N}_{3}$ to have a mass greater than $H_{I}\left(\sim 10^{12-13} \mathrm{GeV}\right)$, implying (from arguments in the previous paragraph) that it is not displaced from its true (late-time) minimum during inflation. The other sneutrinos may or may not be displaced depending on their masses and the magnitude and sign of the coefficient $c_{2}^{k}$. However, the desired baryon asymmetry can only be generated if the heaviest right-handed sneutrino does not get displaced during inflation. Therefore, we will only focus on the heaviest right handed sneutrino and assume that it is not displaced from its true minimum.

Since the heaviest right handed sneutrino $\left(\tilde{N}_{3}\right)$ is naturally expected to settle to its minimum during inflation as argued above, one can integrate out $\tilde{N}_{3}$ and consider the corresponding $L H_{u}$ direction (giving

11 in order to solve the moduli problem, see section 
the lightest left-handed neutrino mass), which becomes a flat-direction as it only gets contributions from supersymmetry breaking and non-renormalizable operators. The lowest dimension operator involving the $L H_{u}$ flat direction is $\frac{\kappa L H_{u} L H_{u}}{M}$ as mentioned in section $\coprod \mathrm{BB}^{2}$. It is worth noting, that even though this operator violates $B-L$, it does preserve $R$-parity. We now turn to the evolution of flat-directions during inflation, focussing on the $L H_{u}$ direction, also denoted by $\phi$, in particular.

The fate of flat directions during inflation also depends on the coefficient of the Hubble induced mass term $\left(c_{3}\right)$. However, since the flat-directions have gauge and yukawa couplings to other matter fields, one also needs to take into account the effect of renormalization (RGE) from the scale at which they are introduced (typically the compactification scale in string compactifications $\sim M_{G U T}, M_{s}$ ) to lower scales $\left(\sim H_{I}\right)$. So, it could happen that RG effects could make the effective mass-squared parameter $\left(m_{\phi}^{\text {eff }}\right)^{2}$ negative at some scale $Q_{c}$ even if $c_{3}$ is positive $\mathcal{O}(1)$ [26]. As argued in [26], the $L H_{u}$ direction is most likely to develop a negative mass-squared parameter for a wide range of $c_{3}$ (both positive and negative) due to RG evolution because of the large top yukawa coupling. The $L H_{u}$ direction has other advantages as well in addition to providing a mechanism for generating the $B-L$ asymmetry and giving rise to viable neutrino masses. Once the $L H_{u}$ direction is displaced, most flat-directions (in the MSSM) are lifted at the renormalizable level so that only the $L H_{u}$ direction needs to be considered for further analysis. Finally, the $L H_{u}$ direction is free from the $Q$-ball problem, to be discussed in section IIIB2. Therefore, the $L H_{u}$ flat-direction is the most natural and robust candidate for generation of the baryon asymmetry.

To summarize, the heavy moduli, the heaviest right handed sneutrino and most flat-directions settle down in their true minima very quickly while the light moduli and the $L H_{u}$ flat direction (corresponding to the lightest left-handed neutrino) are displaced from their minima by a large amount of $\mathcal{O}\left(H_{I}\right)$. For the light moduli, naively one might think that their post-inflationary evolution is fraught with the overshoot problem [27]. However, as was argued in [23, 28, 29], in the presence of matter or radiation (which is true in the present case) the fields can be easily guided towards the global minimum without overshooting. Thus, there is no overshoot problem in this framework. We now study the post-inflationary evolution of the $L H_{u}$ flat-direction in detail.

\section{B. Post-Inflationary Evolution}

Let us study the potential (5) in more detail, with particular attention to the $L H_{u}$ direction and $\tilde{N}_{3}$. The analysis of this section is similar to the one in [7, 8] which was the first to look at the evolution of the $L H_{u}$ flat direction with a gauged $U(1)_{B-L}$ in a systematic manner. However, there are some important differences. The origin of the $U(1)_{B-L}$ breaking and that of the mass of the $U(1)_{B-L}$ gauge boson is different leading to some subtle differences in the analysis; the generation of left-handed and right-handed neutrinos uses a different mechanism leading to different mass-scales; and finally, the embedding of the baryon asymmetry generation mechanism in a complete string framework gives rise to different regions of allowed parameter space relevant for neutrino masses. In the following, we will only outline the important steps here and leave a detailed analysis to appendix C.

12 This could arise by itself ("weinberg" case) and/or by integrating out right-handed neutrinos ("see saw" case). 
The scalar potential for these fields can be schematically written as:

$$
V=V_{\text {susy }}+V_{D}+V_{\text {hubble }}+V_{\text {soft }}
$$

The (normalized) superpotential for the canonically normalized $\hat{L}, \hat{H}_{u}$ and $\hat{N}_{3}$ is given by:

$$
\hat{W}=\hat{h} \hat{N}_{3} \hat{L} \hat{H}_{u}+\hat{M}_{N} \hat{N}_{3} \hat{N}_{3}+W^{\prime}\left(\psi_{i}, \hat{L}, \hat{H}_{u}, \hat{N}_{3}\right)
$$

$\psi_{i}$ stand for fields charged under $U(1)_{B-L}$ (in addition to the MSSM and right handed neutrino fields) with charge $q_{i}$. $W^{\prime}$ contains terms depending on $\psi_{i}$ alone as well as terms containing cross-couplings between $\psi_{i}$ and $\left\{\hat{L}, \hat{H}_{u}, \hat{N}_{3}\right\}$. The mass parameter for $\hat{N}_{3}$ arises when the $B-L$ gauge symmetry is broken. Since $B-L$ is broken at $v_{B-L} \sim M_{s}$, one can write $\hat{M}_{N}$ as $\hat{M}_{N}=\lambda v_{B-L} \sim \lambda M_{s}$ with $\lambda$ a dimensionless coefficient. In the presence of a $U(1)_{B-L}$ gauge symmetry, a non-trivial contribution from the $U(1)_{B-L} D$-term arises, and is given by:

$$
V_{D}=\frac{g_{B-L}^{2}}{2}\left(|\hat{\tilde{N}}|^{2}-|\hat{\tilde{L}}|^{2}+\sum_{i} q_{i} \psi_{i} \partial_{i} K-\left\langle\frac{1}{4 \pi^{2}} \partial_{T_{m}} K\right\rangle\right)^{2}
$$

where $K$ is the Kähler potential. The last term in (10) depends on $T_{m}$ and behaves as an effective Fayet-Iliopoulos (FI) parameter once $T_{m}$ gets a vev (its origin will be explained in section IV). $T_{m}$ is precisely the modulus which appears in the mass parameter for $\hat{\tilde{N}}_{3}$ (see (17)). It is assumed that $T_{m}$ is stabilized by a combination of effects such as higher order corrections to the kähler potential and/or moduli trapping. As explained in the previous section, the mass of the heaviest right handed sneutrino $\left(\hat{M}_{N}\right)$ can naturally be greater than the hubble parameter during inflation $\left(H_{I}\right)$. Thus, $\hat{\tilde{N}}_{3}$ quickly settles down to its minimum during inflation. The minimum is approximately given by:

$$
\left\langle\hat{\tilde{N}}_{3}\right\rangle \approx-\mathcal{O}(1) \frac{\hat{h} \hat{\tilde{L}} \hat{H}_{u}}{\hat{M}_{N}}
$$

The above expression for $\hat{\tilde{N}}_{3}$ can be substituted in (8) to generate a potential for the $L H_{u}$ flat direction, obtained by substituting the neutral components of the $\tilde{L}$ and $H_{u}$ fields with $\phi$. One might worry that the large $D$-term contribution in (10) will destroy the flatness of $\phi$. However, the fields $\psi_{i}$ in (10) will shift in general to make the $D$-term vanish and minimize the potential. This is justified since the curvature around the $D$-term potential is of $\mathcal{O}\left(M_{s}^{2}\right)$ which is much larger than that of the $F$-term potential. This has been shown explicitly in appendix C. Thus, $\phi$ can remain approximately flat and obtain a large expectation value during inflation. After integrating out $\hat{\tilde{N}}_{3}$ and other fields like $\psi_{i}, \bar{\psi}_{i}$, one gets the following expression for the potential for $\phi$ :

$$
\begin{aligned}
V(\phi)= & V_{0}-c_{\phi}^{2} H^{2}|\phi|^{2}+k_{\phi}^{2} H^{2} \frac{|\phi|^{4}}{M_{s}^{2}}+\mathcal{O}(1) \frac{H^{2}|\hat{h}|^{2}|\phi|^{4}}{|\lambda|^{2} M_{s}^{2}}-\mathcal{O}(1)\left(a H \frac{\hat{h}^{2} \phi^{4}}{\lambda M_{s}}+h . c\right)+\mathcal{O}(1) \frac{|\hat{h}|^{4}|\phi|^{6}}{|\lambda|^{2} M_{s}^{2}} \ldots \\
& +\left(m_{\phi}^{2}|\phi|^{2}+\frac{\hat{h}^{2(n-3)} A m_{3 / 2} \gamma \phi^{n}}{n \lambda^{n-3} M_{s}^{n-3}}+c . c\right)+\ldots
\end{aligned}
$$

where $V_{0}$ is the $\phi$-independent contribution, $\left\{c_{\phi}^{2}, k_{\phi}^{2}, a, A\right\}$ are dimensionless coefficients typically of $\mathcal{O}(1)$. “..." stands for higher order terms proportional to powers of $\left(\frac{\hat{h}}{\lambda}\right)$ and $\left(\frac{\hat{h}^{2}}{\lambda}\right)$, among others. We consider the 
case in which $\left(\frac{\hat{h}}{\lambda}\right)$ and $\left(\frac{\hat{h}^{2}}{\lambda}\right)$ are suppressed so that it is possible to neglect those terms. In section IVB, it will be argued that these can be naturally achieved. We will also see later that in order to produce the required baryon asymmetry, one needs a very small value of $\left(\frac{\hat{h}^{2}}{\lambda}\right)$. Thus, terms proportional to $\left(\frac{\hat{h}^{2}}{\lambda}\right)$ are required to be suppressed for consistency.

It is worthwhile to understand the origin of the various terms in (12). The potential for $\phi$ arises from supersymmetry breaking (both hubble induced and hidden sector) and non-renormalizable terms (after integrating out $\hat{\tilde{N}}_{3}$ and $\psi_{i}, \bar{\psi}_{i}$ ). The terms in the second line in (12) are contributions from hidden sector (soft) supersymmetry breaking which are much smaller than the hubble parameter during and just after inflation $^{13}$. Under the above conditions, the minimization of the potential (12) with respect to $|\phi|$ can be simplified:

$$
\begin{aligned}
\frac{\partial V}{\partial|\phi|} & \approx-2 c_{\phi}^{2} H^{2}|\phi|+4 k_{\phi}^{2} H^{2} \frac{|\phi|^{3}}{M_{s}^{2}}=0 \\
\Longrightarrow|\phi|^{2} & \approx \frac{c_{\phi}^{2}}{2 k_{\phi}^{2}} M_{s}^{2}
\end{aligned}
$$

Since $c_{\phi}, k_{\phi}$ are of $\mathcal{O}(1)$ (see appendix C), one has ${ }^{14}$ :

$$
|\phi| \approx \mathcal{O}(1) M_{s}
$$

Even though terms proportional to $\left(\frac{\hat{h}^{2}}{\lambda}\right)$ in (12) are suppressed, as the hubble parameter decreases after inflation, eventually the fourth and fifth terms (proportional to $\left(\frac{\hat{h}^{2}}{\lambda}\right)$ ) in (12) will become comparable to the second and third terms. This will happen when :

$$
H \approx \mathcal{O}(1) \frac{\hat{h}^{2}}{\lambda} M_{s} \equiv H_{0}
$$

Remember that $M_{s}$ is greater than $H_{I}$. Thus, for solution (14) to be consistent, one must have $M_{s}>$ $H_{I}>H>H_{0}$. This is possible since we have $\frac{h^{2}}{\lambda} \ll 1$. It will be argued in section that values of $\frac{h^{2}}{\lambda} \ll 1$ are quite natural in string constructions where the majorana mass of neutrinos arises from string instantons. In the regime $M_{s} \sim v_{B-L}>H_{I}>H>H_{0}$, the dominant contribution to the potential for the phase of $\phi\left(\phi=|\phi| e^{i \theta}\right)$ in (12) is given by:

$$
\begin{array}{r}
V(\hat{\theta}) \approx m_{\hat{\theta}}^{2}|\hat{\theta}|^{2} ; \hat{\theta} \equiv|\phi| \theta \\
\text { where } m_{\hat{\theta}}^{2} \approx \frac{H_{I}}{M}|\phi|^{2} \approx \mathcal{O}(1) \frac{\hat{h}^{2}}{\lambda} M_{s} H<H^{2}
\end{array}
$$

Thus, $\hat{\theta}$ is not settled at the minimum of its potential during inflation and just after inflation. When the hubble parameter drops to $\sim H_{0}$ such that $m_{\hat{\theta}}^{2} \sim H_{0}^{2}$, the hubble $A$-term in the potential can kick $\phi$ in the phase direction providing a torque. This is crucial for generating the baryon asymmetry, as we will now argue.

\footnotetext{
${ }^{13}$ As mentioned earlier, we are considering "natural" high scale inflation with low scale supersymmetry.

${ }^{14}$ One needs $|\phi| \lesssim M_{s}$ for consistency of the solution, see appendix C.
} 


\section{Generation of Lepton Number}

After the end of inflation, the Universe is dominated by the coherent oscillations of the inflaton making it matter dominated. This means that the scale factor $R$ goes like $H^{-2 / 3}$ and $H$ keeps decreasing after inflation. We will assume that the production of lepton number takes place in this epoch, justifying it in the next subsection IIIB2,

After inflation, in the regime $v_{B-L}>H_{I}>H>H_{0},|\phi| \approx v_{B-L} \sim M_{s}$ as shown above. The phase of $\phi$ is displaced from its minimum during this regime. When $H$ drops to values such that $H \sim H_{0}$, the curvature of the potential along the phase direction becomes comparable to $H$, and thus the hubble $A$-term provides a torque to the phase of $\phi$. After this time, $\left(\frac{\hat{h}^{2}}{\lambda}\right)^{2} \frac{|\phi|^{6}}{4 M_{s}^{2}}>$ $\left\{k_{\phi}^{2} H^{2} \frac{|\phi|^{4}}{M_{s}^{2}},|a| H \frac{\hat{h}^{2}}{\lambda} \frac{|\phi|^{4}}{M_{s}} \sin (\arg (a)+4 \arg (\phi))\right\}$ This implies that $|\phi| \approx \sqrt{M H}$ for $H_{0}>H>H_{\text {osc }}$ where $M \equiv \frac{\lambda M_{s}}{\hat{h}^{2}}$. Here, $H_{\text {osc }}$ corresponds to the time when $|\phi|$ starts oscillating about its true minimum. The value of $H_{\text {osc }}$ is determined by a combination of susy breaking effects and thermal effects (more on this in the next subsection [IIB 2). As shown in [7], $|\phi|$ drops as $H^{\alpha}(\alpha \gtrsim 1)$ for $H \lesssim H_{\text {osc }}$.

The lepton number density $\left(n_{L}\right)$ related to $\phi$ is given by:

$$
n_{L}=\frac{1}{2} i\left(\dot{\phi^{*}} \phi-\phi^{*} \dot{\phi}\right)
$$

As already explained above, for $v_{B-L}>H_{I}>H>H_{0}$, the phase of $\phi$ is displaced from its minimum and no lepton number is generated. For $H_{0}>H$, the time evolution of $n_{L}$ in the expanding universe for potential (12) is given by:

$$
\dot{n}_{L}+3 H n_{L} \approx \frac{H}{M} \operatorname{Im}\left(a \phi^{4}\right)+\frac{m_{\phi}}{4 M} \operatorname{Im}\left(A \lambda \phi^{4}\right)+\ldots
$$

The total lepton number $N_{L} \equiv R^{3} n_{L}$ is therefore given by:

$N_{L}(t) \approx \int^{t} d t R^{3}\left[\frac{2 H}{M}|a \| \phi|^{4} \sin (\arg (a)+4 \arg (\phi))+\frac{m_{\phi}}{2 M}|A||\lambda|\left|\phi^{4}\right| \sin (\arg (A)+\arg (\lambda)+4 \arg (\phi))(19)\right.$

For $H_{0}>H>H_{o s c}$, one has:

$$
\begin{gathered}
\frac{R^{3} H|a||\phi|^{4}}{M} \sim|a| M H \sim \frac{|a| M}{t} \\
\frac{\left.R^{3} m_{\phi}|A \lambda \|| \phi\right|^{4}}{M} \sim|A \lambda| m_{\phi} M
\end{gathered}
$$

Also, the argument of the sine function of both terms in (19) oscillates with a frequency $f \sim H$, since $m_{\hat{\theta}} \sim H$ in this regime. Thus, the lepton number at $H \sim H_{\text {osc }}$ is given by:

$$
N_{L}\left(t \sim H_{\text {osc }}^{-1}\right) \sim \mathcal{O}(1)\left(M \log \left(\frac{H_{0}}{H_{\text {osc }}}\right)+\left(m_{\phi} M\right)\left(H_{\text {osc }}^{-1}-H_{0}^{-1}\right)\right)
$$

For later times $\left(H_{\text {osc }}>H\right)$, assuming the most conservative case that $|\phi|$ damps as $H^{\alpha}$ with $\alpha \approx 1$, we have:

$$
\begin{aligned}
\frac{R^{3} H|a||\phi|^{4}}{M} & \sim \frac{H^{3}}{M} \sim \frac{1}{M t^{3}} \\
\frac{R^{3} m_{\phi}|A \lambda||\phi|^{4}}{M} \sim \frac{H^{2} m_{\phi}}{M} & \sim \frac{m_{\phi}}{M t^{2}},
\end{aligned}
$$

implying that lepton number production is strongly suppressed for $H<H_{\text {osc }}$. This means that the total lepton number is fixed when $H \sim H_{\text {osc }}$, giving rise to (21) for the total lepton number. 


\section{Satisfying All Constraints}

The above result is quite interesting; however some implicit conditions need to be satisfied for it to hold. First, early oscillations of $\phi$ have to be avoided so that the lepton asymmetry is not suppressed (this has been implicitly assumed in the previous subsection. Also, as assumed one has to show that the production of lepton asymmetry takes place in the inflaton matter-dominated era, i.e. before the reheating process is completed.

The above constraints can be encapsulated in the following set of conditions:

$$
H_{0}>H_{o s c} \gtrsim \Gamma_{\text {inf }}
$$

$H_{o s c}$ is determined by a combination of thermal effects and supersymmetry breaking effects, as explained in [8]. The dominant supersymmetry breaking effect is just given by the soft mass term for $\phi-m_{\phi} \sim m_{3 / 2}$. Also, although we have assumed the energy density is dominated by the inflaton during the inflaton oscillation era, there is still a dilute plasma in this regime with a temperature given by:

$$
T=\left[\left(T_{R}^{i n f}\right)^{2} m_{p} H\right]^{1 / 4}
$$

where $T_{R}^{\text {inf }}$ is the reheat temperature after inflation. This dilute plasma gives rise to two classes of thermal effects - a) A thermal mass term for $\phi\left(\left(m_{\phi}^{t h}\right)^{2} \sim c_{k} f_{k}^{2} T^{2}\right)$ is induced when the fields which couple to $\phi$ have an effective mass $f_{k}|\phi|<T$, giving rise to a potential contribution $V_{1} \sim\left(m_{\phi}^{\text {th }}\right)^{2}|\phi|^{2}$. b) Another thermal contribution to the potential arises because the $S U(3)$ gauge symmetry remains unbroken along the $\mathrm{LH}_{u}$ direction and down type (s)quarks are also massless along this direction. This gives rise to an effective potential $V \propto g_{s}^{2} T^{4}$. However, because the RG evolution of $g_{s}$ depends on the effective masses $\left(\sim f_{k}|\phi|\right)$ of fields $\psi_{k}$ which are coupled to $\phi$, this implies a potential contribution $V_{2} \sim \alpha_{s} T^{4}\left[\sum_{y_{u}|\phi|>T} \frac{2}{3} T\left(R_{u}\right)\right] \log \left(\frac{|\phi|^{2}}{T^{2}}\right)$.

After inflation the hubble parameter decreases and eventually the negative hubble-induced mass term in the potential for $\phi$ is surpassed by the above contributions, i.e.

$$
H^{2} \lesssim m_{\phi}^{2}+\sum_{f_{k}|\phi|<T} c_{k} f_{k}^{2} T^{2}+\alpha_{s}^{2}(T) \frac{T^{4}}{|\phi|^{2}}
$$

which sets the value of $H_{\text {osc }}$. The second term in (26) gives rise to two sub-cases depending on whether $f_{i}$ is small or large [30]. More precisely, one finds

$$
\begin{aligned}
H_{\text {osc }} & =\max \left[m_{\phi}, H_{i}, \alpha_{s} T_{R}^{\text {inf }}\left(\frac{m_{p}}{M}\right)^{1 / 2}\right] \\
\text { where } H_{i} & \equiv \min \left[\frac{m_{p}\left(T_{R}^{\text {inf }}\right)^{2}}{f_{i}^{4} M^{2}},\left(c_{i}^{2} f_{i}^{4} m_{p}\left(T_{R}^{\text {inf }}\right)^{2}\right)^{1 / 3}\right]
\end{aligned}
$$

where $M \equiv \frac{\lambda}{\hat{h}^{2}} M_{s}$ as before. In order to avoid early oscillations, $H_{0}>H_{o s c}$ is required. From (24) and (26), this implies :

$$
T_{R}^{i n f}<\min \left[\min _{k}\left\{\max \left(\frac{f_{k} M_{s}^{3 / 2}}{c_{k}^{1 / 4} m_{p}^{1 / 2}}, \frac{M_{s}^{3}}{c_{k} f_{k}^{2}\left(m_{p} M^{3}\right)^{1 / 2}}\right)\right\}, \frac{M_{s}^{2}}{\alpha_{s}\left(m_{p} M\right)^{1 / 2}}\right]
$$


If the mass parameter $M \equiv \frac{\lambda}{h^{2}} M_{s}$ in (27) is much larger than $M_{s}$, then it is clear that $H_{o s c}$ is determined by the third term in (27). For this, $\frac{\hat{h}^{2}}{\lambda} \ll 1$ is required. In fact, it will be shown in the coming sections that $\frac{\hat{h}^{2}}{\lambda} \sim 10^{-12} \ll 1$ is required for producing the correct baryon asymmetry, which can be obtained naturally in certain classes of string constructions. Then, for $M_{s} \approx 10^{17} \mathrm{GeV}$ and $\frac{\hat{h}^{2}}{\lambda} \sim 10^{-12}$, the constraint (27) gives rise to $T_{R}^{\text {inf }} \lesssim 10^{11-12} \mathrm{GeV}$. This seems to be quite natural. One also has to check the assumption that the lepton asymmetry is produced during the inflaton oscillation dominated era, implying the condition $H_{\text {osc }}>\Gamma_{\text {inf }}$. Thus, $H_{\text {osc }}>\Gamma_{\text {inf }}$ requires:

$$
T_{R}^{i n f} \lesssim \alpha_{s} \frac{m_{p}^{3 / 2}}{M^{1 / 2}}\left(\frac{90}{\pi^{2} g_{*}}\right)^{1 / 2}
$$

which is again satisfied for $T_{R}^{i n f} \lesssim 10^{11-12} \mathrm{GeV}$ and $\frac{\hat{h}^{2}}{\lambda} \sim 10^{-12}$. Thus, we have shown that the above constraints can be satisfied easily.

Before moving on to computing the final baryon asymmetry, it is worth mentioning that the $L H_{u}$ direction is free from the Q-ball problem [7]. Q-balls are non-topological solitons which arise when the coherent oscillation of a flat-direction is unstable against spatial perturbations. This typically happens when the potential for the flat-direction is flatter than the quadratic potential [31]. If Q-balls are formed, then all charges carried by the flat-direction are absorbed by the Q-ball, hence the baryon asymmetry must be provided by the decay of Q-balls, a situation typically disfavored for various reasons. The supersymmetry breaking mass of the $L H_{u}$ direction, however, has a big contribution from the large top yukawa coupling making the potential for $\phi$ steeper than the quadratic potential, thus avoiding the formation of Q-balls.

\section{The Baryon Asymmetry}

We have seen in the previous subsection that a non-zero lepton number is created during the inflatonoscillation dominated epoch. Since the $L H_{u}$ flat-direction also has a non-zero $B-L$ charge, a non-zero $B-L$ number is also generated. An $\mathcal{O}(1)$ fraction of the $B-L$ number generated above is converted by sphaleron effects to a non-zero baryon number since the $B-L$ number is generated at temperatures much above the electroweak phase transition [32]. More precisely, for a model with extra higgs particles, one has:

$$
N_{B}=\frac{24+4 N_{H}}{66+13 N_{H}}\left(N_{B-L}\right)
$$

where $N_{H}$ is the number of higgs doublets. Here, it has been implicitly assumed that all other new particles are much heavier. For an MSSM-like model with two higgs doublets and in which the sparticles are camparable to the higgs masses, the general expression has been computed in [33]. For our purposes, it will suffice that an $\mathcal{O}(1)$ fraction of $B-L$ number is converted to $B$ number. As long as the condensate decays through $B-L$ conserving interactions after the time it starts oscillating, this baryon number is intact and is insensitive to the details of the decay. This will be assumed to be the case. The baryon asymmetry $\left(\frac{n_{B}}{s}\right)$ computed from above is, however, diluted by other sources of entropy production following inflation. The most natural source of such late-time entropy production is the decay of moduli, alluded to in the Introduction. 
As was explained in section IIA, string compactifications which stabilize the Hierarchy as well as the moduli at the same time generically contain a lightest modulus (moduli) of $\mathcal{O}\left(m_{3 / 2}\right)$. It is quite natural for the lightest moduli $m_{X_{\text {lightest }}}$ to be displaced from their minima (see section (IIA). These moduli start oscillating when the hubble parameter drops to $H \sim m_{X_{\text {light }}}{ }^{15}$. These moduli evolve as pressureless matter and hence their contribution relative to the background radiation (from reheating after inflation) grows rapidly. Hence, they quickly dominate the energy density of the Universe. Also, since these moduli couple to the visible sector by only gravitational (planck suppressed) interactions, they decay quite late, very close to the beginning of BBN. Moduli such as $Y_{i}^{16}$ which may be stabilized at an intermediate scale $\left(H_{I}>M_{Y_{i}}>M_{X_{\text {lightest }}}\right)$ do not affect the result for the final baryon asymmetry since that depends only on the entropy production from moduli which decay last, irrespective of which moduli decay earlier.

The decay of lightest moduli long after inflation produces a lot of entropy and dilutes the baryon asymmetry. Therefore, the final baryon asymmetry can be estimated as:

$$
\begin{aligned}
\frac{n_{B}}{s}(\text { final }) & \approx \frac{N_{B} R^{-3}}{\left(\rho^{\left.X_{\text {lightest }} / T_{R}^{X_{\text {lightest }}}\right)}\right.} \\
& \approx \frac{N_{B} H^{2} T_{R}^{X_{\text {lightest }}}}{3 H^{2} m_{p}^{2}}
\end{aligned}
$$

Using (21), we find for the final baryon asymmetry:

$$
\frac{n_{B}}{s}(\text { final })=\mathcal{O}(1) \frac{M T_{R}^{X_{\text {lightest }}}}{m_{p}^{2}}
$$

where we have used conservatively that $\log \left(H_{0} / H_{\text {osc }}\right)$ is $\mathcal{O}(1)$ in (21) and that the second term in (21) is suppressed. This is consistent with all the constraints in section IIIB2. We are only concerned with the estimate of the baryon asymmetry, and the above result is true up to factors of $\mathcal{O}(1)$. As argued in section IA $m_{X_{\text {light }}} \gtrsim m_{3 / 2}$. In order to produce the desired baryon asymmetry, one requires:

$$
\begin{aligned}
& \frac{n_{B}}{s}(\text { final }) \sim 10^{-10} \\
\Longrightarrow & M \equiv \frac{\lambda M_{s}}{\hat{h}^{2}} \sim 10^{29} \mathrm{GeV}
\end{aligned}
$$

Since $M_{s} \sim v_{B-L} \gtrsim 10^{16} \mathrm{GeV}$ for string constructions consistent with standard gauge unification at $M_{G U T} \sim 10^{16} \mathrm{GeV}$, this implies:

$$
\left(\frac{\hat{h}^{2}}{\lambda}\right) \sim 10^{-12} \text { for } M_{s} \sim 10^{17} \mathrm{GeV}
$$

This is consistent with the analysis in section IIIB for the computation of the lepton number since terms proportional to $\frac{\hat{h}^{2}}{\lambda}$ were assumed to be suppressed in the computation. Note that the requirement of a tiny $\frac{\hat{h}^{2}}{\lambda}$ amounts to having a virtually massless lightest left-handed neutrino $\left(\sim 10^{-16} \mathrm{eV}\right)$. We will argue in section IV that the above requirement for a tiny $\frac{\hat{h}^{2}}{\lambda}$ (and hence a virtually massless lightest left-handed

\footnotetext{
${ }^{15}$ We are ignoring thermal corrections since these are typically quite small for moduli.

16 these correspond to the throat moduli in Type IIB compactifications.
} 
neutrino) is quite natural to obtain in string constructions in which string instantons generate the required couplings. However, before doing that it is important to address issues relating to the moduli(gravitino) problem and the origin and abundance of dark matter within this framework.

\section{Moduli (Gravitino) Problem and Non-thermal Dark Matter}

We first address the moduli and gravitino problems within this framework. It is known that if the displaced light moduli have masses $\gtrsim 10 \mathrm{TeV}$, then their decay reheats the Universe to temperatures above a few $\mathrm{MeV}$ (assuming planck suppressed interactions), allowing BBN to occur successfully. Within gravity mediation, the mass of the lightest modulus (moduli) is comparable to that of the gravitino as explained in section IIA. The precise spectrum depends on model-dependent details. One has to be careful, however, about the overproduction of gravitinos from the decay of the lightest modulus, if kinematically allowed. This could create problems for BBN as well as give rise to too much dark matter from their decays [34]. However, this problem can be elegantly solved if the lightest modulus (scalar field) $X_{0}$ has mass $m_{X_{0}} \approx m_{3 / 2} \gtrsim 10 \mathrm{TeV}$. As explained in section IA , such fields are naturally available since there exist moduli which are not stabilized solely by non-perturbative effects. In addition, constructions in which supersymmetry breaking is triggered by matter scalar fields in a dynamical supersymmetry breaking (DSB) sector, also give rise to $m_{X_{0}} \approx m_{3 / 2}$ [19]. Therefore, the gravitino problem is naturally avoided as the decay of $X_{0}$ to the gravitino is not allowed kinematically. A similar mechanism is operative in the $M$ theory framework studied in [23]. $X_{0}$ decays last and the baryon asymmetry is determined by the reheat temperature of $X_{0}$. As mentioned above, as long as $m_{X_{0}} \approx m_{3 / 2} \gtrsim 10 \mathrm{TeV}, T_{R}^{X_{0}}$ is bigger than a few $\mathrm{MeV}$ allowing BBN to proceed in the usual manner.

Within gauge mediation (presumably embedded in a string compactification), there will again be (geometric) moduli coming from the compactification; however their spectra depends on the concrete embedding and is largely decoupled from low energy phenomenology. In this situation, it is natural to expect that the geometric moduli are reasonably heavier than $\sim 10 \mathrm{TeV}$ implying that they decay well before BBN. However, there is still the scalar partner of the goldstino (which we denote by $S$ ) whose $F$-term breaks supersymmetry. In simple and generic models of gauge mediation, the scalar $S$ is a little heavier than $m_{3 / 2}$ given by $m_{S} \sim m_{3 / 2}\left(\frac{m_{p}}{\Lambda}\right)$, where $\Lambda$ is a high scale present in the kähler potential (see appendix $\mathrm{A}$ for details). Since this scalar has much stronger couplings to the visible sector compared to that of geometric moduli, even for $m_{3 / 2} \lesssim 1 \mathrm{GeV}$ the scalar $S$ could decay before BBN and reheat the Universe to temperatures above a few $\mathrm{MeV}$ [35].

What can be said about the superpartner spectrum and dark matter within this framework? Within gravity mediation, it has been shown that if the modulus which couples to the gauginos is stabilized from non-perturbative effects, then the mass of the gauginos is suppressed relative to $m_{3 / 2}$ [18]. If in addition, the supersymmetry breaking sector is sequestered from the visible sector, then the scalars are also suppressed relative to $m_{3 / 2}$, otherwise not. Therefore, the precise superpartner spectrum will depend on these model-dependent details. It is important to note, however, that even with $m_{3 / 2} \gtrsim 10 \mathrm{TeV}$, it is naturally possible to have superpartners, particularly gauginos, in the sub-TeV range. Within gauge mediation, the gravitino is the LSP $\left(m_{3 / 2} \lesssim \mathrm{GeV}\right)$ and hence the DM candidate. The masses of other superpartners depend on the precise mass scale of the messengers, but can easily take values in the sub-TeV range. 
Finally, we address the issue of the abundance of DM within string compactifications in general and within the framework considered, in particular. As explained in section IA, stabilizing the moduli and generating a stable hierarchy between the electroweak and planck scales at the same time requires that at least some of the moduli are light, close to the TeV scale. Since the Hubble parameter during inflation is typically much larger than the $\mathrm{TeV}$ scale, the light moduli are generically displaced from their minima during inflation and start oscillating when the hubble parameter drops down such that $H \sim M_{\text {moduli }}$. These quickly dominate the energy density of the Universe. Therefore, within string compactifications which stabilize moduli and explain the Hierarchy, the "standard" thermal computation of relic dark matter abundance has to be modified to take the effects of the moduli into account. Since the couplings of visible sector fields are determined by (at least some of) the light moduli, the moduli couple to the visible sector fields and can decay into them.

Within gravity mediation, this means that the light moduli decaying into superpartners will eventually decay to the LSP (DM candidate). The most important such contribution will come from the lightest moduli $X_{0}$. Since $m_{X_{0}} \gtrsim 10 \mathrm{TeV}$, the reheat temperature from decay of $X_{0}$ is $T_{R}^{X_{0}} \sim \sqrt{\Gamma_{X_{0}} m_{p}} \sim$ $\mathcal{O}(1) \mathrm{MeV}$, where $\Gamma_{X_{0}} \sim \frac{m_{X_{0}}^{3}}{m_{p}^{2}}$ is the decay width of $X_{0}$. Since the thermal freeze-out temperature of DM particles $T_{f}^{\chi_{1}} \sim \frac{m_{\chi}}{25} \sim \mathcal{O}(1) \mathrm{GeV}$ is typically much larger than $T_{R}^{X_{0}}$, this means that the DM particles produced from decay of $X_{0}$ never reach thermal equilibrium, thereby giving rise to a nonthermal abundance of dark matter. The thermal abundance of dark matter from the thermal plasma after inflation is greatly diluted by entropy production from decay of the various moduli. Therefore, the non-thermal abundance generically dominates over the thermal one. Scenarios of non-thermal Dark Matter which fit within this framework have been explored in [23, 36].

Within gauge mediation, the situation is quite similar except that the gravitino is the DM candidate and is stable. The decay of geometric moduli before the decay of $S$ will again greatly dilute the thermal abundance of the gravitinos. The scalar $S$ can however, decay directly into gravitinos giving rise to a non-thermal abundance of $\mathrm{DM}^{17}$, similar to that in gravity mediation. This is due to the fact that the reheat temperature resulting from the decay of $S$ is $T_{R}^{S} \gtrsim \mathcal{O}(10) \mathrm{MeV}$ for "reasonable" values of $m_{3 / 2}$ and $\Lambda^{18}$, which is much smaller than the typical freeze-out temperature $T_{f}^{(3 / 2)}$ of gravitinos implying that the gravitinos produced never reach thermal equilibrium.

Depending on the details of the particular construction, the yield of DM particles from the decay of $X_{\text {lightest }}$ could be above or below that of the so-called "critical" density at $T=T_{R}^{X_{\text {lightest }} \text { : }}$

$$
n_{\chi}^{(c)}=\left.\frac{3 H}{\left\langle\sigma_{\chi} v\right\rangle}\right|_{R} ^{X_{l i g h t e s t}}
$$

If the yield is above the critical yield, the DM particles quickly annihilate until they reach the critical density above. On the other hand, if the yield of DM particles is below the critical yield, then the comoving abundance $\left(Y_{\chi} \equiv n_{\chi} / s\right)$ is given by:

$$
Y_{\chi} \sim \frac{B_{X_{\text {lightest }}}^{\chi} n_{\chi}^{(0)}}{\left(T_{R}^{X_{\text {lightest }}}\right)^{3}}
$$

\footnotetext{
${ }^{17}$ If staus are NLSPs, they can also be produced from the decay of $S$ if kinematically allowed. BBN constraints then typically imply an upper bound for the gravitino mass [35]. The precise value is model-dependent.

18 The precise number is model-dependent and depends on details. See appendix $\mathrm{A}$ for more discussion.
} 
In both situations, and both within gravity and gauge mediation, the final relic density depends on the underlying physics determining the coupling of the lightest modulus to visible sector particles. Therefore, the upper bound on the observed relic density serves as an important constraint on the moduli-matter and moduli-gravitino coupling in string constructions. It has been argued that such non-thermal production mechanisms can provide the correct DM abundance in some string frameworks, such as the one in [23].

\section{MICROSCOPICS}

\section{A. $U(1)_{s}$, Anomalies, Gauge Invariance and Brane Instantons}

In this section, we give a brief account of Type II string constructions in which perturbatively absent couplings (forbidden by gauge and global symmetries) can be generated by stringy effects and which have been studied in the literature. Examples of such couplings include yukawa couplings, the $\mu$ parameter, majorana mass terms for neutrinos and certain non-renormalizable operators [25]. In this work, we will primarily be interested in right-handed neutrino majorana masses and the $B-L$ violating "Weinberg" operator leading to neutrino masses.

Within the setup of Type IIB orientifold flux compactifications with D3/D7 branes and orientifold planes (in which moduli stabilization is best understood), semi-realistic matter spectra can be constructed with D3 branes at singularities and/or with D7 branes wrapping supersymmetric four-cycles. Important consistency constraints arise from the requirement of tadpole cancellation ${ }^{19}$. In particular, in order to have chiral spectra from D7-branes, the D7-branes must be equipped with a non-trivial magnetic flux on their world-volume. It is known that a chiral spectrum is anomalous in general. However, it can be shown that the requirement of tadpole cancellation actually guarantees the absence of non-abelian gauge anomalies. The cancellation of pure abelian and mixed abelian-non-abelian(graviton) anomalies is more subtle. The cancellation of these potential anomalies is guaranteed in string theory by the Green-Schwarz mechanism, as follows.

The action for a D7-brane $\left(\mathrm{D} 7_{a}\right)$ contains a Chern-Simons term (among others) of the form:

$$
S_{C S} \sim \int_{\mathbf{R}^{3,1} \times \Sigma_{a}} C_{4} \wedge \mathcal{F} \wedge \mathcal{F}
$$

where $C_{4}$ is the RR 4-form in Type IIB string theory and $\mathcal{F}$ is a two-form. Taking one of the $\mathcal{F}$ along $\Sigma_{a}$ (part of world-volume flux on $\mathrm{D} 7_{a}$ ) and the other $\mathcal{F}$ to be the field strength of the $U(1)_{a}$ gauge field on $\mathrm{D} 7_{a}$, and expanding $C_{4}$ as:

$$
C_{4}=C_{2}^{\alpha} \wedge \omega_{\alpha}+\ldots
$$

gives under certain topological conditions:

$$
S_{C S} \sim \int_{\Sigma_{a}} \omega_{\alpha} \wedge \mathcal{F} \int_{\mathbf{R}^{1,3}} C_{2}^{\alpha} \wedge F_{a} \sim \alpha^{\prime 2} Q_{\alpha}^{a} \int_{\mathbf{R}^{1,3}} C_{2}^{\alpha} \wedge F_{a}
$$

with $Q_{\alpha}^{a}$ an integer-valued topological charge matrix. The $C_{2}^{\alpha} \wedge F_{a}$ coupling leads to two effects - a) it provides a Stuckelberg mass term for the $U(1)_{a}$ gauge field, and b) the shift symmetries of the axions

19 this is bascially a generalization of Gauss's law for fluxes. 
$\left(a^{\alpha} \equiv \operatorname{Im}\left(T^{\alpha}\right)\right.$ ) which are dual to the RR 2-forms $C_{2}^{\alpha}$ are gauged. The gauged shift symmetry of the axions gives rise to a $D$-term for the corresponding $U(1) \mathrm{s}$ as in (10).

The $U(1)_{a}$ gauge fields which remain massless lie in the kernel ${ }^{20}$ of the matrix $Q_{a}^{\alpha}$. These $U(1)_{s}$ are always anomaly free. For example, in realistic string constructions $U(1)_{Y}$ must always be massless; so it must lie in the kernel. On the other hand, massive $U(1)_{a}$ gauge fields could be either anomalous or anomaly free depending on the details. For anomalous $U(1) \mathrm{s}$, there appears an additional term in the effective action - $\int_{\mathbf{R}^{1,3}} a^{\alpha} \operatorname{tr}\left(F^{\alpha} \wedge F^{\alpha}\right)$ which precisely cancels the mixed abelian-non-abelian gauge(graviton) anomalies alluded to above. The above coupling is not present for non-anomalous $U(1) \mathrm{s}$ since these are already anomaly free by definition. Non-anomalous massive $U(1) \mathrm{s}$ are quite interesting as they provide a way to reduce the gauge symmetry at low energies without the Higgs mechanism. A natural example of such a massive anomaly-free $U(1)$ is $U(1)_{B-L}$. This can naturally generate majorana neutrino masses and the $B-L$ violating weinberg operator, as we will see below.

Focussing on string constructions with a $U(1)_{B-L}$ gauge symmetry, we are interested in the case when the details of the compactification lead to a structure where the two forms $C_{2}^{\alpha}$ (or their duals $a^{\alpha}$ ) couple to the $U(1)_{B-L}$ gauge field in such a way as to provide a Stuckelberg mass term for the gauge field. The massive $U(1)_{B-L}$ still survives at low energies as a perturbative global symmetry. Therefore, majorana neutrino masses, among other operators, are forbidden at the perturbative level. However, nonperturbative effects may exist which violate this global symmetry to a discrete subgroup ${ }^{21}$ and generate these operators. Such a non-perturbative effect actually does exist in string theory; it is provided by (euclidean) brane instantons. Because the effective perturbative $U(1)_{B-L}$ global symmetry arising at low energies is secretly gauged in string theory, the operator induced by the brane instantons must respect the underlying gauge invariance. This implies, in particular, that the non-perturbatively generated majorana mass parameter $\left(M_{N}\right)$ should transform under $U(1)_{B-L}$ in such a way as to make the operator $M_{N} N N$ $U(1)_{B-L}$ gauge invariant. This mechanism is quite general and can formally occur in Type IIA, Type IIB, Heterotic and $M$ theory constructions. Most of the work related to model-building in this regard is done in Type IIA constructions [38] ${ }^{22}$ where it has been shown that there exists a large set of models satisfying the various criteria required for the above mechanism to work. Since Type IIA constructions are related by T-duality to Type IIB constructions, we expect a large set of models incorporating the above mechanism to exist within Type IIB constructions as well. Assuming this to be the case, we will keep working within the framework of Type IIB compactifications with D3/D7 branes ${ }^{23}$.

We briefly describe the microscopic mechanism by which the brane instanton generates the relevant operators and the conditions which need to be satisfied. In computing the spacetime interaction mediated by the instanton, one has to integrate over the instanton zero modes. The brane instanton relevant in Type IIB compactificatiosn are E3 instantons wrapping supersymmetric four-cycles in the compact space and invariant under the orientifold projection. In order to contribute to the effective superpotential, the instanton must have the right structure of zero modes. Generically, one only has universal bosonic zero modes, those corresponding to the position of the instanton. The fermionic zero modes are much more

\footnotetext{
20 The kernel of a matrix $\mathbf{Q}$ is the set of all eignevectors $\mathbf{x}$ for which $\mathbf{Q} \mathbf{x}=0$.

${ }^{21}$ In many cases, it turns out that the discrete subgroup is none other than the usual $R$-parity [38].

22 see [39] for some work in local Type IIB constructions.

23 This is because moduli stabilization is best understood in this setup.
} 
important though. The uncharged (under the 4D gauge group) fermionic zero modes have to satisfy certain conditions to contribute to the superpotential [25]. We will focus on (chiral) charged fermionic zero modes which are much more interesting and relevant. Microscopically, these charged fermionic zero modes arise from open strings at the intersection of the E3 instanton $E$ with spacetime-filling magnetized D7-branes $D 7_{i}$ (and their orientifold images $D 7_{i}^{\prime}$ ) present in the construction. The net number of these zero modes is given by $I_{E i}-I_{E i^{\prime}}$, where $I_{E i}$ is the intersection number between $E$ and $D 7_{i}$. In order to saturate the integration over the charged fermionic zero modes, the spacetime interaction must therefore contain insertions of charged (in particular under $\left.U(1)_{s}\right) 4 \mathrm{D}$ fields, giving rise to a superpotential of the following general form:

$$
W \sim e^{-S_{E 3}} \phi_{1} \phi_{2} \ldots \phi_{n}
$$

The action of the instanton is proportional to the volume of the four-cycle which it wraps. This is measured by a kähler modulus $\left(T^{E} \equiv \tau^{E}+i a^{E}\right)$, whose imaginary part is an axion $a^{E}$ which shifts as described in the previous section since the D7-branes are magnetized.

$$
\begin{aligned}
\operatorname{Re}\left(\mathrm{S}_{\mathrm{E}_{3}}\right) & \sim \frac{2 \pi}{g_{s}} \frac{\operatorname{Vol}\left(\Sigma_{4}\right)}{l_{s}^{4}} \equiv 2 \pi \tau^{E} \\
\operatorname{Im}\left(\mathrm{S}_{\mathrm{E}_{3}}\right) & \sim 2 \pi a^{E}
\end{aligned}
$$

From the structure of zero modes and Stuckelberg couplings of the type $\int C_{2}^{\alpha} \wedge F_{a}$, one can show that under $U(1)_{X}=\sum_{i} U(1)_{i}$ :

$$
\begin{aligned}
e^{-S_{E 3}} & \rightarrow e^{-i \sum_{i}\left(I_{E i}-I_{E i^{\prime}}\right) \Lambda_{i}} e^{-S_{E 3}} \\
\phi_{1} \phi_{2} \ldots \phi_{n} & \rightarrow e^{i \sum_{i}\left(I_{E i}-I_{E i^{\prime}}\right) \Lambda_{i}} \phi_{1} \phi_{2} \ldots \phi_{n}
\end{aligned}
$$

Thus the E3-instanton transforms under $U(1)_{X}$ in such a way as to precisely cancel the transformation of the charged matter fields, making the superpotential in (39) $U(1)_{X}$ gauge invariant. From the low energy effective field theory point of view however, the $U(1)_{X}$ appears as a global symmetry which is broken by the $E 3$ instanton ${ }^{24}$.

\section{B. Application to Neutrinos}

We can now apply the above formalism to the special case of majorana neutrino masses and the weinberg operator with $U(1)_{X}=U(1)_{B-L}$. This has been done in the literature. The zero mode structure required to obtain the two operators is different for the two cases [38]. Hence, two different kinds of E3 instantons contribute to the two operators. Depending on the details of the construction, one or both kinds of instantons may contribute to neutrino masses. We will look at both of them in detail.

When the structure of zero modes satisfies a certain condition [38], one gets the following operator:

$$
\begin{aligned}
W_{M} & =M_{N}^{a b} N_{R}^{a} N_{R}^{b} \\
\text { where } M_{N}^{a b} & =m_{p} \sum_{r} d_{r}^{a} d_{r}^{b} e^{-S_{E 3}^{(M), r}}
\end{aligned}
$$

${ }^{24}$ generally to a discrete subgroup. 
where $d_{r}^{a, b}$ are model-dependent coefficients of $\mathcal{O}(1)$. In general, many instantons (with the same zero mode structure) contribute to the majorana mass parameter. The summation in (42) illustrates that fact. The flavor structure of (42) is such that having three or more instantons gives rise to three non-zero eigenvalues. Since the volumes wrapped by these instantons will generically differ by $\mathcal{O}(1)$, the three mass eigenvalues of the matrix $M_{N}^{a b}$ will generically be hierarchical, as stated in section [IIA 39$]$. This will thus lead to the following left-handed neutrino masses:

$$
M_{L}^{a b}(\text { seesaw })=\left\langle H_{u}^{0}\right\rangle^{2}\left(\mathbf{h}^{\mathbf{T}} \mathbf{M}_{\mathbf{N}}^{-1} \mathbf{h}\right)^{a b}
$$

The Weinberg operator, which requires a different structure of zero modes [38], is given by:

$$
\begin{aligned}
W_{W} & =\kappa \frac{L H_{u} L H_{u}}{m_{p}} \\
\Longrightarrow M_{L}^{a b} \text { (weinberg) } & =\frac{\left\langle H_{u}^{0}\right\rangle^{2}}{m_{p}} \sum_{r} c_{r}^{a} c_{r}^{b} e^{-S_{E 3}^{(W), r}}
\end{aligned}
$$

Again, many different instantons (with the same zero mode structure) could contribute to the left-handed neutrino masses and naturally lead to small left-handed neutrino masses.

As mentioned above, depending on the details one or both kinds of instantons may contribute to the neutrino masses. It could also happen that one may dominate the other. Note however, that in string vacua with $N_{R}$ 's massless at the perturbative level, one definitely requires instantons to make the $N_{R}$ 's massive at a phenomenologically acceptable level. Therefore, here we will assume that both kinds of E3 instantons discussed above exist. In the explicit string constructions considered in [38], such examples were found in large number ${ }^{25}$. We will consider both cases where the instanton generating majorana masses dominates over the one generating the weinberg operator and vice versa, and argue that it is naturally possible to obtain the required value of $\left(\frac{\hat{h}^{2}}{\lambda}\right)$ in both cases. Also, since we are primarily interested in the baryon asymmetry, we will mostly focus on the lightest left-handed neutrino mass. In section VI, however, a very interesting possibility will be briefly described in which one has $\mathcal{O}(\mathrm{TeV})$ right handed neutrinos consistent with high-scale affleck-dine leptogenesis and the moduli problem. Potential consequences of these for Dark Matter and the LHC will also be briefly discussed.

Before moving on to the details, we would like to comment on the flavor structure of the neutrino mass matrix. The detailed flavor structure is model-dependent; however some general aspects of the flavor structure within this context have been studied in [40] where it was shown that it is possible to incorporate a flavor structure for the neutrino mass matrix consistent with observations with reasonable assumptions. Since we are not interested in the detailed flavor structure for our purposes, for concreteness and simplicity we will assume the "normal hierarchy" case with sequential dominance [41]. This is also natural from a theoretical point of view since the different instanton contributions are naturally hierarchical as argued above. In fact, within the paradigm of sequential dominance, it is quite natural to have only two instantons dominantly give rise to the observed neutrino masses and mixings making the lightest left-handed neutrino virtually massless [40, 41]. This is precisely what is required for achieving a large enough baryon asymmetry within the framework studied here. It has to be kept in mind though that other flavor structures are also possible. Those can also be probably incorporated within this framework.

${ }^{25}$ Large number of models were found after satsifying a relaxed constraint on the symmetries of the instantons which can easily occur in the presence of fluxes [38]. 


\section{Microscopic Constraints to obtain a small $\frac{h^{2}}{\lambda}$}

In this subsection, we will look at the computation of the lightest neutrino mass in detail. The expression for the left-handed neutrino mass matrix was given in (43) and (44). However, those expressions are not canonically normalized. One has to be careful in properly normalizing the matter fields such as to give rise to a canonical kinetic term for these fields. This is especially important within supergravity since the Kähler potential for these fields is non-canonical in general. In general, if the (un-normalized) superpotential is given by:

$$
W=M_{\alpha \beta} \phi_{\alpha} \phi_{\beta}+Y_{\alpha \beta \gamma} \phi_{\alpha} \phi_{\beta} \phi_{\gamma}+\frac{\kappa_{\alpha \beta \gamma \delta}}{m_{p}} \phi_{\alpha} \phi_{\beta} \phi_{\gamma} \phi_{\delta}+\ldots
$$

then the canonically normalized quantities in $W$ above are [42]:

$$
\begin{aligned}
\hat{M}_{\alpha \beta} & =e^{K / 2} M_{\alpha \beta}\left(\tau_{m}\right) \\
\hat{Y}_{\alpha \beta \gamma} & =e^{K / 2} \frac{Y_{\alpha \beta \gamma}\left(U_{m}\right)}{\left(\tilde{K}_{\alpha} \tilde{K}_{\beta} \tilde{K}_{\gamma}\right)^{1 / 2}\left(\tau_{m}, U_{m}\right)} \approx e^{K / 2} \frac{Y_{\alpha \beta \gamma}\left(U_{m}\right)\left(g_{\alpha}^{-1} g_{\beta}^{-1} g_{\gamma}^{-1}\right)\left(U_{m}\right)}{\left(\tilde{K}_{\alpha}^{0} \tilde{K}_{\beta}^{0} \tilde{K}_{\gamma}^{0}\right)^{1 / 2}\left(\tau_{m}\right)} \text { (no sum) } \\
\hat{\kappa}_{\alpha \beta \gamma \delta} & =e^{K / 2} \frac{\kappa_{\alpha \beta \gamma \delta}\left(U_{m}\right)}{\left(\tilde{K}_{\alpha} \tilde{K}_{\beta} \tilde{K}_{\gamma} \tilde{K}_{\delta}\right)^{1 / 2}\left(\tau_{m}, U_{m}\right)} \approx e^{K / 2} \frac{\kappa_{\alpha \beta \gamma}\left(U_{m}\right)\left(g_{\alpha}^{-1} g_{\beta}^{-1} g_{\gamma}^{-1} g_{\delta}^{-1}\right)\left(U_{m}\right)}{\left(\tilde{K}_{\alpha}^{0} \tilde{K}_{\beta}^{0} \tilde{K}_{\gamma}^{0} \tilde{K}_{\delta}^{0}\right)^{1 / 2}\left(\tau_{m}\right)} \text { (no sum) }
\end{aligned}
$$

In the above, we have shown the moduli dependence of the various parameters and have used the fact that the kähler metric $\tilde{K}_{\alpha}$ for matter fields $\phi_{\alpha}$ can be factorized as $\tilde{K}_{\alpha}\left(\tau_{m}, U_{m}\right)=\tilde{K}_{\alpha}^{0}\left(\tau_{m}\right) g_{\alpha}\left(U_{m}\right)^{26}[43]$. Applying the above formulas to the majorana mass operator and the weinberg operator, one gets the following contributions to the left-handed neutrino mass:

$$
\begin{aligned}
\hat{M}_{\left(\nu_{a b}\right)}^{L}(\text { seesaw }) & =\mathcal{O}(1) \frac{\left\langle H_{u}^{0}\right\rangle^{2}}{m_{p}} \frac{e^{K / 2}}{\tilde{K}_{L}^{0} \tilde{K}_{H_{u}}^{0} \tilde{K}_{N_{3}}^{0}} \frac{\left(\mathbf{h}_{\mathbf{D}}^{T} \mathbf{h}_{\mathbf{D}}\right)}{\left(\sum_{r} d_{a}^{r} d_{b}^{r} e^{-S_{E 3}^{(M), r}}\right)} \\
\hat{M}_{\left(\nu_{a b}\right)}^{L}(\text { weinberg }) & =\mathcal{O}(1) \frac{\left\langle H_{u}^{0}\right\rangle^{2}}{m_{p}} \frac{e^{K / 2}}{\tilde{K}_{L}^{0} \tilde{K}_{H_{u}}^{0}}\left(\sum_{r} c_{a}^{r} c_{b}^{r} e^{-S_{E 3}^{(W), r}}\right)
\end{aligned}
$$

Here, we have used the fact that generically $\tilde{K}_{\alpha}\left(\tau_{m}, U_{m}\right)=\mathcal{O}(1) \tilde{K}_{\alpha}^{0}\left(\tau_{m}\right)$ and have also used a diagonal neutrino yukawa coupling for simplicity $\left(\mathbf{h} \approx \mathbf{h}_{\mathbf{D}}\right)$. One can further simplify the above expressions if one assumes that the various instantons contributing to the operators above are hierarchical, which we have argued to be quite natural. Thus, the sum over the instantons in both operators in (47) above is dominated by only one instanton. Using the fact that typically $c_{a}^{r}, d_{a}^{r}=\mathcal{O}(1)$, this gives rise to the following expression for the lightest left-handed neutrino mass:

$$
\begin{aligned}
\hat{M}_{\left(\nu_{1}\right)}^{L}(\text { seesaw }) & =\mathcal{O}(1) \frac{\left\langle H_{u}^{0}\right\rangle^{2}}{m_{p}} \frac{e^{K / 2}}{\tilde{K}_{L}^{0} \tilde{K}_{H_{u}}^{0} \tilde{K}_{N_{3}}^{0}}\left(h_{D}^{1}\right)^{2}\left(e^{S_{E 3}^{(M)}}\right) \\
\hat{M}_{\left(\nu_{1}\right)}^{L}(\text { weinberg }) & =\mathcal{O}(1) \frac{\left\langle H_{u}^{0}\right\rangle^{2}}{m_{p}} \frac{e^{K / 2}}{\tilde{K}_{L}^{0} \tilde{K}_{H_{u}}^{0}}\left(e^{-S_{E 3}^{(W)}}\right)
\end{aligned}
$$

\footnotetext{
${ }^{26}$ For simplicity, we have also assumed that the kähler metric is roughly diagonal, i.e. $\tilde{K}_{\bar{\alpha} \beta} \approx \tilde{K}_{\alpha} \delta_{\alpha \beta}$. This will not change the main conclusion in the analysis.
} 
For later purposes, it is useful to write down expressions for the dependence of the string scale $M_{s}$, the kähler potential and the kähler metric on the volume (kähler moduli). These are given by [44]:

$$
\begin{aligned}
M_{s} & =\frac{m_{p}}{\mathcal{V}^{1 / 2}} \\
K & =-2 \log \mathcal{V}+\ldots \\
\tilde{K}_{\phi} & =\tau_{m}{ }^{-1 / 2} \mathcal{O}(1)=\mathcal{V}^{-1 / 3} \mathcal{O}(1)
\end{aligned}
$$

where for the kähler metric we have assumed for simplicity that all four-cycles in the Calabi-Yau are roughly of the same size. Since we are interested in models with standard gauge unification, a natural choice is $M_{G U T} \lesssim M_{s} \sim 10^{17} \mathrm{GeV}$, implying that $\mathcal{V}^{1 / 2}=\mathcal{O}(10)$. Also, for the supergravity approximation to hold, we will require that the moduli are stabilized at values greater than unity, but still respecting the constraint that $\mathcal{V}^{1 / 2}=\mathcal{O}(10)$. We will use all this in our subsequent analysis.

\section{Majorana Operator Domination (see-saw case)}

We will first consider the case where the see-saw contribution dominates over that of weinberg one. From (47), this requires:

$$
\left(h_{D}^{1}\right)^{2} \gg e^{-\left(S_{E 3}^{(M)}+S_{E 3}^{(W)}\right)} \tilde{K}_{N_{3}}^{0}
$$

In addition, in order to obtain the correct baryon asymmetry (33) in this case, one requires:

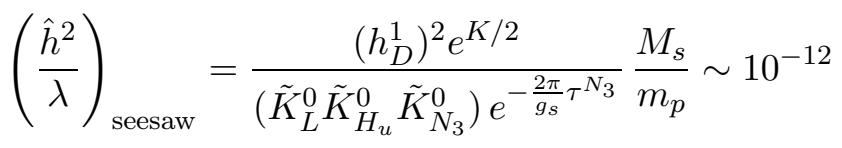

Here we have used the fact $\hat{M}_{N_{3}}=e^{K / 2} e^{-2 \pi \tau_{N_{3}}} m_{p}=\hat{\lambda} M_{s}$, implying $\hat{\lambda}=\frac{e^{K / 2} e^{-2 \pi \tau_{N_{3}}} m_{p}}{M_{s}}$. Using (499), one gets:

$$
\left(h_{D}^{1}\right)^{2} e^{2 \pi \tau_{N_{3}}} \sim 10^{-12} \mathcal{V}^{1 / 2} \mathcal{O}(1)
$$

The value of $\tau_{N_{3}}$ is constrained by the requirement that the right-handed sneutrino $\hat{\tilde{N}}_{3}$ is not displaced from its original minimum in order to produce a non-zero lepton number as argued in appendix $B$, Conservatively, this means that $M_{N_{3}}$ is larger than the hubble parameter during inflation, i.e.

$$
\begin{aligned}
\hat{M}_{\tilde{N}_{3}} & \approx M_{N_{3}}=\mathcal{O}(1) e^{K / 2} e^{-2 \pi \tau_{N_{3}}} m_{p} \gtrsim H_{I} \\
\Longrightarrow \frac{e^{-2 \pi \tau_{N_{3}}}}{\mathcal{V}} m_{p} & \gtrsim H_{I} \approx 10^{12}-10^{13} \mathrm{GeV}
\end{aligned}
$$

This implies that $\tau_{N_{3}}$ be close to (but greater than) unity for $\mathcal{V}^{1 / 2}=\mathcal{O}(10)$. This is still consistent with the supergravity approximation.

The un-normalized yukawa couplings $h_{D}$ arise from multiply wrapped world-sheet instantons and are given by products of Jacobi theta functions [45]. These can be well approximated as $h_{D}^{i j k} \approx \mathcal{O}(1) e^{-\eta^{i j k} 4 \pi U_{m}}$ where $\eta^{i j k}=\mathcal{O}(1)$. These yukawas depend on the complex structure moduli $\left(U_{m}\right)$ in Type IIB string theory which are stabilized by bulk fluxes and can take a wide range of values depending on the fluxes. 
From (52) and (53), one finds that $\left(h_{D}^{1}\right)^{2} \gtrsim 10^{-15}$. This can be naturally obtained by $U_{m}=\mathcal{O}(1)$ (but greater than unity) ${ }^{27}$.

Also, the condition (50) that the majorana operator dominates the weinberg operator gives rise to a constraint on $\tau_{W}$. Using (52), the condition (50) gives:

$$
\begin{gathered}
e^{-2 \pi \tau_{W}} \ll 10^{-10} \\
\Longrightarrow \tau_{W} \gtrsim 3.6
\end{gathered}
$$

which can again be satisfied naturally. Finally, we have to check that for the above choice of parameters the quantity $\left(\frac{\hat{h}}{\lambda}\right)$ is suppressed as has been assumed in the analysis of section IIIB, One finds that:

$$
\left(\frac{\hat{h}}{\lambda}\right)_{\text {see saw }}=\frac{\left(h_{D}^{1}\right)}{\left(\tilde{K}_{L}^{0} \tilde{K}_{H_{u}}^{0} \tilde{K}_{N_{3}}^{0}\right)^{1 / 2} e^{-2 \pi \tau^{N_{3}}}} \frac{M_{s}}{m_{p}} \lesssim 10^{-3}
$$

which is adequately suppressed.

\section{Weinberg Operator Domination}

We now turn to the other case. This gives:

$$
\left(h_{D}^{1}\right)^{2} \ll e^{-\left(S_{E 3}^{(M)}+S_{E 3}^{(W)}\right)} \tilde{K}_{N_{3}}
$$

In order to obtain the correct baryon asymmetry in this case, one requires:

$$
\begin{aligned}
\left(\frac{\hat{h}^{2}}{\lambda}\right)_{\text {weinberg }} & =\frac{e^{K / 2}}{\tilde{K}_{L} \tilde{K}_{H_{u}}} e^{-2 \pi \tau_{W}} \frac{M_{s}}{m_{p}} \sim 10^{-12} \\
\text { or } \frac{e^{-2 \pi \tau_{W}}}{\mathcal{V}^{5 / 6}} & \sim 10^{-12} ; \quad \text { using (499) } \\
\Longrightarrow \tau_{W} & \approx 3.5-4
\end{aligned}
$$

The requirement for the majorana mass of $\tilde{N}_{3}$ is the same as in the previous case:

$$
\begin{aligned}
\hat{M}_{\tilde{N}_{3}} \approx \hat{M}_{N_{3}} & =\mathcal{O}(1) e^{K / 2} e^{-2 \pi \tau_{N_{3}}} m_{p} \gtrsim H_{I} \\
e^{-2 \pi \tau_{N_{3}}} & \gtrsim 10^{-4}
\end{aligned}
$$

again leading to $\tau_{N_{3}}$ close to (but greater than) unity. Using (157) and (58), the condition (56) for weinberg operator domination gives:

$$
\left(h_{D}^{1}\right)^{2} \ll \frac{10^{-16}}{\mathcal{V}^{1 / 2}} \approx 10^{-17} \text { using (49) }
$$

So, using $h_{D}^{i j k} \approx \mathcal{O}(1) e^{-\eta^{i j k}} 4 \pi U_{m}$ where $\eta^{i j k}=\mathcal{O}(1)$, one finds that (59) requires $U_{m} \gtrsim 1.5$. Finally, one can estimate:

$$
\left(\frac{\hat{h}}{\lambda}\right)_{\text {weinberg }} \sim \frac{\left(h_{D}^{1}\right)}{\left(\tilde{K}_{L}^{0} \tilde{K}_{H_{u}}^{0} \tilde{K}_{N_{3}}^{0}\right)^{1 / 2} e^{-2 \pi \tau_{N_{3}}}} \frac{M_{s}}{m_{p}} \ll 10^{-4}
$$

$\overline{27}$ The $\mathrm{O}(1)$ factors at various places allow multiple ways of satisfying the constraint. 
which is consistent with our assumption.

Thus, we find that the range of the values of the stabilized moduli in both cases are different from each other. Nevertheless, both sets of ranges are perfectly natural to obtain from perturbative string compactifications which are consistent with the supergravity approximation and a compactification scale close to the unification scale $M_{G}$. Finally, it is important to remember that it could happen that both contributions to neutrino masses are comparable to each other. A much bigger parameter space for the stabilized values of the moduli opens up in the general case, although the analysis is more complicated. Qualitatively however, the analyses in the previous subsections show that it is possible to obtain the required baryon asymmetry with moduli fixed in the supergravity regime and a small neutrino yukawa coupling.

\section{OTHER STRING/ $M$ THEORY COMPACTIFICATIONS}

In this work, we have mostly focussed on Type IIB compactifications. However, as long as the relevant microscopic criteria are satisfied, the mechanism described in this paper could work in other kinds of string/ $M$ theory compactifications which stabilize the moduli and generate the Hierarchy at the same time. One such example in which it is naturally possible to achieve the above is $M$ theory compactifications studied in [12]. Examples in other corners of string/ $M$ theory may exist as well. Within $M$ theory compactifications, it was shown in [12] that with some reasonable assumptions, strong gauge dynamics in the hidden sector can stabilize all the bulk moduli as well as generate the Hierarchy in a natural manner in a de Sitter (dS) vacuum. Supersymmetry breaking is mediated to the visible sector by gravity. The moduli spectra can then be computed reliably and it turns out that the lightest moduli have masses $M_{X_{\text {lightest }}} \approx m_{3 / 2}$ which decay close to (but earlier than) BBN generating a large amount of entropy [23]. Therefore, the microscopic criteria required in order to produce the correct baryon asymmetry by the Affleck-Dine mechanism in this case are precisely the same as in the Type II case. Also, since $m_{3 / 2}$ can naturally be greater than $\sim 10 \mathrm{TeV}$ and supersymmetry breaking is mediated by gravity, there is no moduli (gravitino) problem within this framework. It is known that $M$ theory compactified on a singular seven dimensional space with $G_{2}$ holonomy is dual, in different regions of its moduli space, to both heterotic string theory on an appropriate Calabi-Yau [52] and Type IIA string theory on another appropriate Calabi-Yau with D6-branes and O6-planes [53]. Since many examples of heterotic and Type IIA string models with an $S M \times U(1)_{B-L}$ gauge group and an MSSM-like chiral spectrum (with right-handed neutrinos) exist in literature, this means that in principle this is possible in the $M$ theory constructions as well. Finally, masses, yukawa couplings and non-renormalizable terms like the Weinberg operator in $M$ theory arise from membrane instantons connecting the various superfields

[52, 53]. So, the appropriate zero-mode structure has to be satisfied in order for the instantons to contribute to the relevant terms in the superpotential, similar to that in Type II constructions. It would be extremely interesting to have explicit constructions satisfying the above criteria. 


\section{POTENTIAL CONSEQUENCES FOR OBSERVABLE PHYSICS}

It would be very interesting to look for possible signals in particle physics and cosmology experiments in order to test the ideas proposed in this paper. Although precise details are model-dependent, one can still make general observations which are nevertheless quite interesting. As already pointed out in section IIID, the framework generically predicts a non-thermal mechanism for the production of Dark Matter. One consequence of this is that DM candidates with a much larger annihilation cross-section ${ }^{28}$, compared to that required for a standard thermal relic abundance, are allowed [23]. This is essentially because the final relic abundance is set by the physics of the late-decaying scalar. This result is quite intriguing in the sense that a DM interpretation of recent results from DM indirect detection experiments like PAMELA and ATIC also require much larger annihilation cross-sections. If the DM interpretation survives, it might be taken as a possible hint for non-thermal Dark Matter ${ }^{29}$.

Within this framework, a very interesting possibility could exist in which one could have $\mathcal{O}(\mathrm{TeV})$ righthanded neutrinos compatible with the production of baryon number at times much earlier than that of the electroweak phase transition. Moreover, the $\mathcal{O}(\mathrm{TeV})$ right-handed neutrino will not be accompanied by a low energy $U(1)_{B-L}$. Although such a light right-handed neutrino is not a definite prediction of the framework, it is quite interesting and hence worth some attention because it has consequences for Dark Matter as well as the LHC.

As already explained, the right-handed neutrino masses are naturally hierarchical since they are suppressed exponentially relative to one another. Therefore, it is quite possible to have the lightest righthanded neutrino to be light, near the electroweak scale. Depending upon which operator (majorana or weinberg) is dominantly responsible for the heaviest left-handed neutrino mass (for which there is an experimental upper bound), different parameter spaces are available for the stabilized values of the moduli such that the lightest right-handed neutrino is around the electroweak scale ${ }^{30}$. If both operators are comparable, a much bigger parameter space opens up. The requirement that the lightest right-handed neutrino be around the electroweak scale implies:

$$
\begin{aligned}
\hat{M}_{N_{1}}=\mathcal{O}(1) \frac{e^{-2 \pi \tau_{N_{1}}} m_{p}}{\mathcal{V}} & \sim 10^{2} \mathrm{GeV} \\
\Longrightarrow \tau_{N_{1}} & \approx 5.1
\end{aligned}
$$

Note that the above could be true irrespective of which operator, majorana or weinberg, is responsible for the lightest left-handed neutrino mass $M_{\nu_{1}}$ and hence the baryon asymmetry $n_{B} / s$. Thus, it is possible ${ }^{31}$ for the baryon asymmetry to be produced much before the electroweak phase transition while still having an electroweak scale right-handed neutrino.

If such a light right-handed neutrino exists, it has interesting consequences for the LHC as well as Dark Matter. To understand this better, it is useful to write the relevant interactions. Assuming that the neutrino yukawas are roughly diagonal as before, we can write the relevant interactions containing

\footnotetext{
28 by a factor of upto $\mathcal{O}(1000)$.

${ }^{29}$ Other explanations are also possible.

30 remember we have assumed sequential dominance of right-handed neutrinos.

31 note that this is a natural possibility but not a concrete prediction.
} 
the lightest right-handed neutrino as:

$$
\begin{aligned}
W & \supset \hat{h}_{3} \hat{N}_{1} \hat{L}_{3} \hat{H}_{u}+\hat{M}_{N_{1}} \hat{N}_{1} \hat{N}_{1} \\
\mathcal{L}_{\text {soft }} & \supset \tilde{m}^{2} \hat{\tilde{N}}_{1}^{\dagger} \hat{\tilde{N}}_{1}+\left(\hat{A}_{3} \hat{h}_{3} \hat{\tilde{N}}_{1} \hat{\tilde{L}}_{3} \hat{H}_{u}+\frac{1}{2} B \hat{M}_{N_{1}} \hat{\tilde{N}}_{1} \hat{\tilde{N}}_{1}+\text { h.c. }\right)
\end{aligned}
$$

The first line above corresponds to supersymmetric interactions while the second line corresponds to soft supersymmetry breaking interactions.

An important consequence of the above lagrangian is that the lightest sneutrino can be much lighter than the lightest right-handed neutrino. The reason is that the $2 \times 2$ sneutrino mass-squared matrix is modified because of the right-handed diagonal mass-squared contribution $\left(M_{R}^{2} \equiv \hat{M}_{N_{1}}^{2}+\tilde{m}^{2}\right)$ and the off-diagonal $A$-term contribution in (62) (when the higgs gets a vev). RG running from high-scale to low-scale can have important effects as well. This leads generically to a situation in which the two mass eigenstates are split by a large amount, leading to an eigenstate with suppressed mass and couplings compared to that in the MSSM. The $Z$-width constraint (for sneutrinos lighter than $m_{Z}$ ) can also be satisfied if the lightest eigenstate is predominantly $\hat{\tilde{N}}_{1}$. Thus, the possibility of sneutrino dark matter opens up quite naturally within gravity mediation. A scenario with similar features for the right-handed neutrino and sneutrino has been studied in [46] but from a very different theoretical perspective. However, the phenomenological consequences are quite similar for right-handed neutrinos and sneutrinos. From (62), we see that there is generically also a $B-L$ violating $B$-term in the soft lagrangian. If for some reason, this $B$-term is suppressed compared to the $B-L$ conserving mass terms for the sneutrinos, it will split the CP-odd and CP-even states by a small amount. This could give rise to an inelastic sneutrino dark matter scenario which could explain the positive signal at DAMA [47] while being consistent with the negative results of other direct detection experiments such as CDMS, XENON, ZEPLIN, KIMS and CRESST. Such a possibility was pointed out in [48]. Of course, within the theoretical framework considered in this paper, one has to come up with a natural way of obtaining the correct splitting. This is left for future work.

Depending on the precise pattern of masses of the right-handed neutrino and the sneutrinos, there could also be a wide variety of possibilities for collider physics. For instance, if the right-handed neutrino is really light - lighter than the SM-like higgs, then the higgs could preferentially decay to right handed neutrinos which in turn decay to six particles in the final state [49]. Even if such a decay is kinematically not allowed, the higgs could decay to $\tilde{\nu}_{1} \tilde{\nu}_{1}^{*}$ since the lightest sneutrino can naturally be quite light as explained in the previous paragraph. This could give rise to an invisibly decaying higgs which would make it hard to discover it at the LHC by standard methods, although other channels may open up which still make it possible [50]. The presence of unsuppressed $A$-terms can give non-standard charged higgs decays, with $H^{ \pm}$decaying to $\tilde{L}_{L} \tilde{N}_{1}$. It could also lead to production of the light $\left(\tilde{\nu}_{1}\right)$ and heavy $\left(\tilde{\nu}_{2}\right)$ sneutrinos which could decay either visibly or invisibly leading to different signatures. In certain cases, $\tilde{\nu}_{2}$ could decay to $\tilde{\nu}_{1}+h$ with a large branching fraction providing an interesting new way to produce higgs particles in cascade decays which could be comparable to standard channels. Thus, having light right-handed neutrino and sneutrinos opens up a wealth of possibilities at the LHC. In order to make more concrete predictions however, one needs to specify an explicit pattern of the other superpartners such as the gauginos, squarks and the sleptons and then look at the possible consequences in a systematic manner. 
It is important to note that the lightest neutrino mass in this framework is virtually massless. This property can also lead to observable signals. For example, the rate for neutrinoless double beta decay depends on the effective mass of the electron-type neutrino $m_{\nu_{e e}}$ (for majorana neutrinos). If the light neutrinos follow a hierarchical pattern as in the present framework with the lightest of them being virtually massless, it is possible to bound $m_{\nu_{e e}}$ from both sides. This could lead to a potential observation in the future. This is similar to the arguments in [51].

\section{CONCLUSIONS}

The standard paradigm of cosmological evolution consists of a radiation dominated era resulting from the decay of the inflaton which lasts until well after the end of Big-Bang Nucleosynthesis (BBN). Within this paradigm, DM particles are created from the thermal plasma and eventually freezeout giving rise to a thermal relic abundance which has to be compared with observations. Most models trying to explain the baryon asymmetry also work within the above paradigm. However, as we have argued in this paper, within classes of realistic string compactifications which stabilize the moduli as well as generate the Hierarchy at the same time, the standard paradigm is no longer applicable. This is because in such compactifications, there generically exists at least one light modulus which is comparable to the gravitino mass (within a few orders of magnitude). Since the Hubble parameter during inflation $H_{\text {inf }}$ is typically much larger than the $\mathrm{TeV}$ scale for natural inflationary models, this modulus is generically displaced during inflation. When the hubble parameter drops down to the mass of the modulus, the modulus starts to oscillate and quickly dominates the energy density of the Universe. Since the modulus couples only very weakly to the matter fields, it decays close to BBN, generating a large entropy and diluting any pre-existing baryon asymmetry. Thus, most mechanisms explaining the baryon asymmetry have to take this crucial feature into account.

In this work, we have argued that it is still possible to generate the required baryon asymmetry in a natural manner within classes of realistic string compactifications with a minimal extension of the MSSM below the unification scale (only right-handed neutrinos) and satisfying certain microscopic criteria. This is achieved by a period of Affleck-Dine leptogenesis from the $L H_{u}$ flat-direction shortly after the end of inflation, which has been studied earlier in the literature. In this work, we have embedded the above mechanism (with some important differences compared to the original proposals) in a complete framework motivated from string theory and addressed all relevant issues starting from the end of inflation to the beginning of BBN.

Since the $\mathrm{LH}_{u}$ flat-direction is obtained after integrating out heavy right-handed majorana neutrinos; therefore the issue of neutrino masses is intricately connected to the baryon asymmetry. In fact, generating the correct baryon asymmetry requires that the lightest left-handed neutrino mass is virtually massless, of $\mathcal{O}\left(10^{-16} \mathrm{eV}\right)$. It is quite interesting that a virtually massless lightest left-handed neutrino is allowed by data. We have also argued that it is possible to generate such a light left-handed neutrino mass naturally from string instantons (satisfying certain constraints). The moduli and gravitino problems can be naturally solved in this framework both within gauge and gravity mediation, although in a different manner. Because of the decay of heavier moduli before that of the lightest one, any thermal abundance of DM particles (both in gravity and gauge mediation) is greatly diluted. Hence, the relic abundance of DM particles comes from the direct decay of moduli. Since the decay widths depend on the moduli- 
matter and moduli-gravitino couplings, the upper bound on the relic abundance provides an important constraint on these couplings. Since these couplings are themselves determined in terms of the structure of the kähler potential among other things, this may provide important insights about the effective action arising in a particular framework. It is important to note that it is naturally possible to have superpartners, particularly gauginos, in the sub-TeV range in this framework (both within gravity and gauge mediation). The framework also leads to some broad potential signals for particle physics and cosmology. A non-thermal origin of Dark Matter, which is quite natural within this framework, may be crucial to explain the recent results of indirect detection experiments like PAMELA and ATIC. Also, a light right-handed (s)neutrino at around the electroweak scale is naturally allowed (although it is not predicted). This could have very interesting consequences both for Dark Matter direct detection and the LHC. Finally, the fact that the lightest neutrino is virtually massless could potentially lead to a positive signal at neutrinoless double-beta decay experiments.

Each of the microscopic criteria required have separately been shown to be satisfied for explicit constructions and are mutually compatible with each other; it is therefore expected that these criteria could also be satisfied simultaneously by a sizable fraction within the sub-landscape of realistic string vacua, providing a solution to all the above problems in a natural manner. Nevertheless, it would be extremely convincing and useful if one could have explicit string constructions satisfying most (if not all) microscopic criteria listed in this paper. This is technically challenging at present, but could be achieved in the near future.

It is also worth mentioning that given the existence of light moduli decaying close to BBN in a stringmotivated framework, there could be other ways of generating the required baryon asymmetry compared to the one described here. For example, one could imagine a scenario in which both the baryon asymmetry and Dark Matter are produced from the decays of a heavy scalar field dominating the energy density of the Universe before BBN. Such models have been studied in [54], and it may be possible to embed them (again maybe with some differences) naturally in a string theoretic framework [55]. In principle, this mechanism of producing the baryon asymmetry is distinguishable from the mechanism studied in this paper. For instance, this may be possible in the presence of light right-handed (s)neutrinos because of their special signatures for the LHC and DM. It would be extremely interesting to come up with other ways of distinguishing the different mechanisms with experimental observables.

\section{Acknowledgments}

PK would like to particularly thank Lawrence Hall for very helpful discussions. PK is also thankful to Michael Lennek, Gordon Kane and Scott Watson for useful comments and suggestions. The research of PK is supported in part by the US Department of Energy. Finally, PK would like to thank the Aspen Center for Physics and the TH Unit at CERN for their hospitality where part of the research was conducted. 


\section{APPENDIX A: LIGHTEST “MODULUS" IN GAUGE MEDIATION}

In this section, we will study the lightest scalar field ("modulus") in gauge mediation. In accordance with our philosophy the gauge mediation model should be embedded in a string compactification, although from a conceptual perspective since gauge mediation models work at parametrically low energies, the details of the compactification and string embedding should not have much effect on low energy physics. Thus, it is reasonable to expect that a string embedding will stabilize the geometric moduli with masses much above the $\mathrm{TeV}$ scale implying that they will decay much before BBN even if some of them are displaced from their minima during inflation. However, there are other scalar fields (which we also denote by moduli by a slight abuse of notation) whose effects have to be taken into account. Many models of gauge mediation exist in the literature, and the precise results for the masses of light scalar fields will depend on model-dependent details. However, we would like to argue that there are some generic features which exist in a large class of gauge mediation models.

Here we study a simple scheme of gauge mediation at low energies which was argued to be quite generic [35, 56]:

$$
\begin{aligned}
W & =W_{0}+\mu^{2} S-\kappa S \bar{f} f \\
K & =|f|^{2}+|\bar{f}|^{2}+|S|^{2}-\frac{|S|^{4}}{\Lambda^{2}}+\ldots
\end{aligned}
$$

where $S$ is the goldstino superfield which parameterizes supersymmetry breaking, $f, \bar{f}$ are the messenger fields charged under both the visible and hidden gauge groups, $\Lambda$ is a mass scale at which other massive fields have been integrated out and $W_{0}$ is the constant piece of the superpotential required to obtain a vanishing (tiny) cosmological constant. The presence of $W_{0}$ breaks $R$-symmetry. A good string embedding should microscopically account for this constant piece $W_{0}{ }^{32}$. Even though one is studying a scheme for gauge mediation, one still needs to compute the potential within supergravity as this is the low energy theory obtained from string compactifications preserving $\mathcal{N}=1$ SUSY. Within supergravity, the potential arising from the above superpotential and the kähler potential has a long-lived metastable supersymmetry breaking minimum at [57]:

$$
\begin{gathered}
\langle S\rangle \sim \frac{\Lambda^{2}}{m_{p}} ;\langle f\rangle=\langle\bar{f}\rangle=0 ; \\
\left\langle F_{S}\right\rangle \approx \mu^{2} \Longrightarrow m_{3 / 2} \sim \frac{\mu^{2}}{m_{p}}
\end{gathered}
$$

For $\Lambda \gtrsim 10^{13} \mathrm{GeV}$, this minimum is stable and the masses of the scalars $S$ and the messengers are given by [57]:

$$
m_{S} \sim \frac{\mu^{2}}{\Lambda} ; \quad m_{f, \bar{f}} \sim \frac{\Lambda^{2}}{m_{p}}
$$

An upper limit for $\Lambda$ is $m_{p}$. In order to find out which of the scalars is lighter, one has to impose the constraint that the mediation of supersymmetry breaking by gauge interactions dominates compared to

32 after stabilizing the moduli. 
that by gravitational interactions (our original assumption). This leads to an upper bound on $m_{3 / 2}$, $m_{3 / 2} \lesssim \mathcal{O}(1) \mathrm{GeV}$. Using $m_{p} \gtrsim \Lambda \gtrsim 10^{13} \mathrm{GeV}$ from above and conservatively using the upper bound for $m_{3 / 2}$, one finds that $m_{f, \bar{f}}>m_{S}$. Thus, $S$ is generically the lightest scalar in generic models of gauge mediation with a mass:

$$
m_{S} \sim \frac{\mu^{2}}{\Lambda} \sim m_{3 / 2}\left(\frac{m_{p}}{\Lambda}\right)
$$

Regarding possible values of $\Lambda, \Lambda \sim M_{G U T} \sim 10^{16} \mathrm{GeV}$ seems to be both theoretically and phenomenologically [35] interesting implying that $m_{S} \sim 10^{2} m_{3 / 2}$, although other values in the above range are presumably allowed. Thus, the lightest scalar in generic gauge mediation models is a few orders of magnitude above $m_{3 / 2}$. Finally, since $S$ is coupled much more strongly to the visible sector than a gravitationally coupled scalar, it can easily reheat the Universe to temperatures above a few $\mathrm{MeV}$ even for much smaller masses.

\section{APPENDIX B: WHAT IF $\hat{\tilde{N}}_{3}$ IS DISPLACED DURING INFLATION?}

In this section, we will argue that if $\hat{\tilde{N}}_{3}$ is displaced from its true minimum during inflation, then it is not possible to generate the required baryon asymmetry. During and after inflation, the dominant (mass-squared) contributions to the potential for $\hat{\tilde{N}}_{3}$ are given by:

$$
V=\left(M_{N}^{2}+c_{N}^{2} H^{2}+m_{0}^{2}\right)\left|\hat{\tilde{N}}_{3}\right|^{2}+\left[\left(B+b_{N} H\right) M_{N} \hat{\tilde{N}}_{3} \hat{\tilde{N}}_{3}+\text { h.c. }\right]+\ldots
$$

The parameters $b$ and $B$ can be taken to be real without loss of generality. The above mass matrix can be diagonalized to give mass eigenstates:

$$
\begin{aligned}
& \hat{\tilde{N}}_{R}=\frac{1}{\sqrt{2}}\left(\hat{\tilde{N}}_{3}+\hat{\tilde{N}}_{3}^{*}\right) \\
& \hat{\tilde{N}}_{I}=\frac{-i}{\sqrt{2}}\left(\hat{\tilde{N}}_{3}-\hat{\tilde{N}}_{3}^{*}\right)
\end{aligned}
$$

with the following eigenvalues:

$$
M_{R, I}^{2}=\left(M_{N}^{2}+c_{N} H^{2}\right) \pm(B+b H) M_{N}
$$

The situation above is quite different compared to that with flat-directions because here one has renormalizable $L$ violating interactions (the $B$ term) in contrast to those present in the case of flat-directions. The $B$ term makes the mass eigenstates non-degenerate, so they will oscillate independently with different frequencies when $H \sim M_{N}$. Therefore, the lepton number created during these oscillations will oscillate in general, in contrast to that for flat-directions. Depending on whether the hubble-induced $B$-term $b$ is $\mathcal{O}(1)$ or suppressed (maybe due to a symmetry), one might hope that it is possible to transfer this lepton number generated for certain ranges of $\Gamma_{N}$. This range is determined in terms of of $B$ and $b[58]$. However, it turns out that even though lepton number can be stored in the oscillations of $\hat{\tilde{N}}_{R}$ and $\hat{\tilde{N}}_{I}$ for certain ranges of $\Gamma_{N}$, it is still not possible to transfer the lepton asymmetry to the (s)leptons. This is because the coupling of the sneutrino to left-handed (s)leptons also violates lepton number (due to the 
$B$-term). A simple way to see this is to write the relevant interaction in the mass eigenstate basis:

$$
\begin{aligned}
-\mathcal{L}_{i n t}= & \hat{\tilde{N}}_{3}\left(\hat{h}_{1} \hat{L} \hat{\tilde{\tilde{H}}}_{u}+\hat{h}_{1}^{*} M_{N} \hat{\tilde{\tilde{L}}} \hat{H}_{u}{ }^{*}+A \hat{h}_{1} \hat{\tilde{L}} \hat{H}_{u}\right)+h . c . \\
= & \frac{\hat{h}_{1}}{\sqrt{2}} \hat{\tilde{N}}_{R}\left[\hat{L} \hat{\tilde{\tilde{H}}}_{u}+\left(A+M_{N}\right) \hat{\tilde{L}} \hat{H}_{u}\right]+h . c+ \\
& i \frac{\hat{h}_{1}}{\sqrt{2}} \hat{\tilde{N}}_{I}\left[\hat{L} \hat{\tilde{\tilde{H}}}_{u}+\left(A-M_{N}\right) \hat{\tilde{L}} \hat{H}_{u}\right]+h . c
\end{aligned}
$$

From (B4), it is clear that the decay widths to (s)leptons and anti-(s)leptons are the same, and hence no asymmetry can be generated. Therefore, it is crucial for $\hat{\tilde{N}}_{3}$ to not be displaced from its minimum for the desired baryon asymmetry to be generated.

\section{APPENDIX C: TECHNICAL DETAILS FOR COMPUTING THE LEPTON NUMBER}

In this section, we will study the potential for the flat-direction $\phi$ during and after inflation in detail. As explained in section \A, for concreteness we assume that the visible sector at around the compactification scale $M_{s}$ (which we assume to be close to the string scale and the GUT scale and the same as the $B-L$ breaking scale from section $)^{33}$ consists of the gauge group $G=S M \times U(1)$, while the matter sector consists of that of the MSSM with three right-handed neutrinos and possibly other (vector-like) exotic fields. These exotic fields typically get massive at $M_{s}$. Some of these exotics could be charged under $U(1)_{B-L}$. This is assumed to be the case. The superpotential, written schematically in (9), is given in detail by:

$$
\hat{W}=\hat{h} \hat{N}_{3} \hat{L} \hat{H}_{u}+\hat{M}_{N} \hat{N}_{3} \hat{N}_{3}+\eta X\left(\psi \bar{\psi}-M_{s}^{2}\right)
$$

Here $\psi, \bar{\psi}, X$ are MSSM singlets and $\psi, \bar{\psi}$ are assumed to be charged (oppositely) under $U(1)_{B-L}$. The third term in (C1) above just provides one possible mechanism to provide a mass of $\mathcal{O}\left(M_{s}\right)$ for $\psi, \bar{\psi}, X$, so its precise form is not crucial for us as long as that is achieved. What is important is the fact that there are fields charged under $U(1)_{B-L}$ which get a mass when $B-L$ is broken. The full potential is given by:

$$
\begin{aligned}
V= & V_{D}+V_{\text {susy }}+V_{\text {hubble }}+V_{\text {soft }} \\
= & \frac{g_{B-L}^{2}}{2}\left(|\hat{\tilde{N}}|^{2}-|\hat{\tilde{L}}|^{2}+q \psi \partial_{\psi} K-q \bar{\psi} \partial_{\bar{\psi}} K-\frac{1}{4 \pi^{2}}\left\langle\partial_{T_{G}} K\right\rangle\right)^{2}+ \\
& \left|\mu \hat{H}_{u}\right|^{2}+\left|\hat{h} \hat{\tilde{L}} \hat{H}_{u}\right|^{2}++\left|\mu \hat{H}_{d}+\hat{h} \hat{N} \hat{L}\right|^{2}+\left|\hat{h} \hat{\tilde{N}} \hat{H}_{u}\right|^{2}+|\eta X \psi|^{2}+|\eta X \bar{\psi}|^{2}+\left|\eta\left(\psi \bar{\psi}-M_{s}^{2}\right)\right|^{2}+ \\
& 3 H^{2}\left(\sum_{Y}\left(b_{Y}^{\prime}\right)|Y|^{2}\right)-H\left(\sum_{Y} c_{Y} Y W_{Y}+\text { h.c. }\right)+V_{\text {soft }}
\end{aligned}
$$

Here $K$ is the Kähler potential for the moduli and matter fields (see (2)), $T_{m}$ is the kähler modulus whose axionic partner shifts under $U(1)_{B-L}$. Since we have assumed that $T_{m}$ is stabilized by effects such as higher order corrections to $K$ or by moduli trapping, the third term in (ㄹㄹ $)$ gives rise to an effective $F I$

${ }^{33}$ Although the GUT scale $M_{G}$ is less than the typical string scale $M_{s}$ by a factor of a few, threshold corrections can account for the discrepancy. 
parameter, $\xi_{\text {eff }} \equiv-\frac{Q_{m}}{4 \pi^{2}}\left\langle\partial_{T_{m}} K\right\rangle$ where $Q_{m}$ is a topological charge. The magnitude of the $F I$ parameter is naturally of $\mathcal{O}\left(M_{s}\right)$ as will be argued at the end of this section. The terms in the third line in (C2) arise from hubble induced supersymmetry breaking during and after inflation; $b_{Y}^{\prime}$ and $c_{Y}$ are typically of $\mathcal{O}(1) . \quad Y$ stands for all the relevant fields $\left\{X, \psi, \bar{\psi}, \hat{\tilde{N}}_{3}, \hat{\tilde{L}}, \hat{H}_{u}, \hat{H}_{d}\right\}$. The contribution from ordinary hidden sector supersymmetry breaking $V_{\text {soft }}$ is much smaller than the other terms, hence it is not written explicitly.

The potential ( $\mathrm{C2}$ ) is quite complicated. In order to make progress, it is convenient to integrate out heavy fields. This is also relevant because field with masses equal to or greater than the hubble parameter settle down at the bottom of the potential and track their respective minima during the subsequent evolution. To this end, we will be interested in the region of field space in which:

$$
\begin{aligned}
|\mu| & \ll H \lesssim \hat{M}_{N}<|\psi| \lesssim M_{s} \\
\hat{h}|\phi| & \ll \hat{M}_{N}
\end{aligned}
$$

where we have introduced the $L H_{u}$ flat-direction $\phi$ defined as:

$$
\hat{\tilde{L}}^{T}=\frac{1}{\sqrt{2}}(\phi 0) ; \hat{H}_{u}^{T}=\frac{1}{\sqrt{2}}\left(\begin{array}{ll}
0 & \phi
\end{array}\right)
$$

We will see that the solutions obtained are consistent with the above. In this region of field space, one finds that $\bar{\psi}, X, \hat{\tilde{N}}_{3}$ and $\hat{H}_{d}$ get heavy masses from the $F$-term contribution to the potential, i.e. from $V_{\text {susy }}+V_{\text {hubble }}+V_{\text {soft }}$ in (C2). Solving the equations of motion for these fields, their vacuum-expectationvalues (vevs) are respectively:

$$
\begin{aligned}
\langle\bar{\psi}\rangle & \approx \frac{M_{s}^{2}}{\psi} ;\langle X\rangle \ll M_{s} ;\left\langle H_{d}\right\rangle \ll M_{s} \\
\left\langle\hat{\tilde{N}}_{3}\right\rangle & \approx-\frac{\hat{h} \hat{\tilde{L}} \hat{H}_{u}}{\hat{M}_{N}}\left(\frac{\left(1-\mathcal{O}(1) \frac{H}{\hat{M}_{N}}\right)}{\left(1+\frac{\hat{h}^{2}\left(\left|\hat{H}_{u}\right|^{2}+|\hat{\tilde{L}}|^{2}\right)}{\left|\hat{M}_{N}\right|^{2}}+\mathcal{O}(1) \frac{H^{2}}{\left|\hat{M}_{N}\right|^{2}}\right)}\right) \\
& \approx-\mathcal{O}(1) \frac{\hat{h} \hat{\tilde{L}} \hat{H}_{u}}{\hat{M}_{N}}
\end{aligned}
$$

in accordance with the restrictions on the field-space above. In the above minimum, $X$ and $\bar{\psi}$ get masses of $\mathcal{O}\left(M_{s}\right), \hat{\tilde{N}}_{3}$ gets a mass of $\mathcal{O}\left(\hat{M}_{N}\right)$ and $\hat{H}_{d}$ gets a mass of $\mathcal{O}(H)$. After integrating out these fields, and neglecting terms proportional to $\mu$ and $V_{\text {soft }}{ }^{34}$, the potential (C2), now in terms of $\psi$ and $\phi$, is given by:

$$
\begin{aligned}
V \approx & \frac{g_{B-L}^{2}}{2}\left[\mathcal{O}(1)\left|\frac{\hat{h} \phi^{2}}{\hat{M}_{N}}\right|^{2}-\frac{1}{2}|\phi|^{2}+q \frac{\left|M_{s}\right|^{4}}{|\psi|^{2}}-q|\psi|^{2}-\frac{N_{F}}{4 \pi^{2}}\left\langle\partial_{T_{G}} K\right\rangle\right]^{2}+\hat{h}^{2}|\phi|^{4}+ \\
& \mathcal{O}(1) \frac{|\hat{h}|^{4}|\phi|^{6}}{\left|\hat{M}_{N}\right|^{2}}+3\left(b_{\phi}^{\prime}\right) H^{2}|\phi|^{2}+\mathcal{O}(1)\left(3 b_{N}^{\prime}+1\right) H^{2} \frac{|\hat{h}|^{2}|\phi|^{4}}{\left|\hat{M}_{N}\right|^{2}}+3\left(b_{\psi}^{\prime}\right) H^{2}|\psi|^{2}+ \\
& 3\left(b_{\bar{\psi}}^{\prime}\right) H^{2} \frac{M_{s}^{4}}{|\psi|^{2}}-\mathcal{O}(1) H\left(\frac{\hat{h}^{2} \phi^{4}}{\hat{M}_{N}}+\text { h.c. }\right)
\end{aligned}
$$

${ }^{34}$ since these are much smaller than other terms 
The $D$-term contribution in (C6) can vanish naturally by a shift of $\psi$. Requiring a vanishing $D$-term is justified since the curvature around the minimum of the $D$-term potential is of $\mathcal{O}\left(M_{s}^{2}\right)$, which is much larger than the curvature $\left(\mathcal{O}\left(H^{2}\right)\right)$ of the $F$-term potential. This guarantees that $\phi$ remains an approximate flat-direction and gets a large vev, as we will see below. From ([C6), the vev of $|\psi|$ is given by:

$$
\begin{aligned}
|\psi|^{2} & =M_{s}^{2}\left[\left(1-\frac{\beta}{4}\right)^{1 / 2}-\frac{\beta}{4}\right] \\
\text { where } \beta M_{s}^{2} & =\left(\frac{1}{2}|\phi|^{2}-\xi_{\text {eff }}-\mathcal{O}(1) \frac{|\hat{h}|^{2}|\phi|^{4}}{\left|\hat{M}_{N}\right|^{2}}\right)
\end{aligned}
$$

$\beta$ has to satisfy the constraint that the quantity $\left(1-\frac{\beta}{4}\right)$ in the square root in (C7) is positive, implying that $\beta<4$. As will be shown self-consistently, $\beta$ can in fact be naturally smaller than unity, so that one can expand the expression for $|\psi|$ in (C7) in powers of $\beta / 4$. This implies that after substituting the expression for $|\psi|$ in (C7) in (C2), one gets the following potential for $\phi$ :

$$
\begin{aligned}
V \approx & \mathcal{O}(1) \frac{|\hat{h}|^{4}|\phi|^{6}}{\left|\hat{M}_{N}\right|^{2}}+3\left(b_{\phi}^{\prime}\right) H^{2}|\phi|^{2}+\mathcal{O}(1)\left(3 b_{N}^{\prime}+1\right) H^{2} \frac{|\hat{h}|^{2}|\phi|^{4}}{\left|\hat{M}_{N}\right|^{2}}-\mathcal{O}(1) H\left(\frac{\hat{h}^{2} \phi^{4}}{\hat{M}_{N}}+h . c .\right)+ \\
& 3 H^{2} M_{s}^{2}\left(b_{\psi}^{\prime}+b_{\bar{\psi}}^{\prime}\right)+\frac{9}{8} H^{2} M_{s}^{2} \beta\left(b_{\bar{\psi}}^{\prime}-b_{\psi}^{\prime}\right)+\frac{3}{128} H^{2} M_{s}^{2} \beta^{2}\left(19 b_{\bar{\psi}}^{\prime}-b_{\psi}^{\prime}\right) \\
\text { or, } V \approx & -\frac{9}{8} H^{2}\left(b_{\bar{\psi}}^{\prime}-b_{\psi}^{\prime}\right) \xi_{\text {eff }}+\frac{3}{128} \frac{H^{2}}{M_{s}^{2}}\left(19 b_{\bar{\psi}}^{\prime}-b_{\psi}^{\prime}\right) \xi_{\text {eff }}^{2}+\left(3\left(b_{\phi}^{\prime}\right)+\frac{9}{16}\left(b_{\bar{\psi}}^{\prime}-b_{\psi}^{\prime}\right)-\frac{3}{128} \frac{\xi_{\text {eff }}}{M_{s}^{2}}\left(19 b_{\bar{\psi}}^{\prime}-b_{\psi}^{\prime}\right)\right) H^{2}|\phi|^{2} \\
& +\mathcal{O}(1) H^{2}|\phi|^{4}\left(\frac{|\hat{h}|^{2}}{|\lambda|^{2} M_{s}^{2}}\left[2+3 b_{N}^{\prime}+b_{\psi}^{\prime}-b_{\bar{\psi}}^{\prime}+\frac{3}{64} \frac{\xi_{\text {eff }}}{M_{s}^{2}}\left(19 b_{\bar{\psi}}^{\prime}-b_{\psi}^{\prime}\right)\right]+\frac{3}{512} \frac{\left(19 b_{\bar{\psi}}^{\prime}-b_{\psi}^{\prime}\right)}{M_{s}^{2}}\right) \\
& -\mathcal{O}(1) H\left(\frac{\hat{h}^{2} \phi^{4}}{|\lambda| M_{s}}+h . c .\right)+\mathcal{O}(1) \frac{|\hat{h}|^{4}|\phi|^{6}}{|\lambda|^{2} M_{s}^{2}}-\mathcal{O}(1) \frac{3}{128}\left(19 b_{\bar{\psi}}^{\prime}-b_{\psi}^{\prime}\right) \frac{H^{2}}{M_{s}^{2}} \frac{|\hat{h}|^{2}|\phi|^{6}}{|\lambda|^{2} M_{s}^{2}} \\
& +\mathcal{O}(1) \frac{3}{128}\left(19 b_{\bar{\psi}}^{\prime}-b_{\psi}^{\prime}\right) \frac{H^{2}}{M_{s}^{2}} \frac{|\hat{h}|^{4}|\phi|^{8}}{|\lambda|^{4} M_{s}^{4}}
\end{aligned}
$$

where we have used $H \lesssim \hat{M}_{N}$ and $\hat{M}_{N} \sim \lambda M_{s}$. From section IIIC, one requires a small $\left(\frac{\hat{h}^{2}}{\lambda}\right)$ to get the desired baryon asymmetry. In addition, we will require a small $\left(\frac{\hat{h}}{\lambda}\right)$ as well. It has been argued in sections IVC 1 and IVC2 this this can be naturally obtained. This will turn out to lead to our initial condition $\hat{h}|\phi| \ll \hat{M}_{N}$. Therefore, the leading order potential will be considerably simplified:

$$
V \approx V_{0}+\left(3\left(b_{\phi}^{\prime}\right)+\frac{9}{16}\left(b_{\bar{\psi}}^{\prime}-b_{\psi}^{\prime}\right)-\frac{3}{128} \frac{\xi_{e f f}}{M_{s}^{2}}\left(19 b_{\bar{\psi}}^{\prime}-b_{\psi}^{\prime}\right)\right) H^{2}|\phi|^{2}+\mathcal{O}(1) H^{2}|\phi|^{4}\left(\frac{3}{512} \frac{\left(19 b_{\bar{\psi}}^{\prime}-b_{\psi}^{\prime}\right)}{M_{s}^{2}}\right)(\mathrm{C} 9)
$$

where all terms proportional to $\left(\frac{\hat{h}}{\lambda}\right)$ and $\left(\frac{\hat{h}^{2}}{\lambda}\right)$ arise at subleading order. $V_{0}$ stands for terms which do not depend on $\phi$. In order to get a large vev, the mass-squared for $|\phi|$ in (C9) has to be negative. As argued in section ЏII, this is quite naturally possible for $b_{\phi}^{\prime}, b_{\psi}^{\prime}, b_{\bar{\psi}}^{\prime} \sim \mathcal{O}(1)$ and $\xi_{\text {eff }} \lesssim M_{s}^{2}$. Hence this will be assumed to be the case. Minimizing the potential with respect to $|\phi|$, one gets:

$$
|\phi|^{2} \approx \mathcal{O}(1) \frac{\left(-3\left(b_{\phi}^{\prime}\right)-\frac{9}{16}\left(b_{\bar{\psi}}^{\prime}-b_{\psi}^{\prime}\right)+\frac{3}{128} \frac{\xi_{e f f}}{M_{s}^{2}}\left(19 b_{\bar{\psi}}^{\prime}-b_{\psi}^{\prime}\right)\right)}{\left(\frac{3}{256}\left(19 b_{\bar{\psi}}^{\prime}-b_{\psi}^{\prime}\right)\right)} M_{s}^{2} \equiv \frac{c_{\phi}^{2}}{2 k_{\phi}^{2}} M_{s}^{2}
$$

(using the notation in section (IIIB) 
we see that $|\phi| \sim M_{s}$ is naturally allowed. We now check the self-consistency of our solutions. $\mu \ll$ $H \lesssim \hat{M}_{N}$ just reflects our starting expectations about low-scale supersymmetry and high scale inflation together with a large right-handed neutrino mass $\hat{M}_{N}$. From the solution for $|\phi|$ above, we have $\frac{\hat{h}|\phi|}{\hat{M}_{N}} \approx$ $\frac{\hat{h}}{\lambda} \frac{|\phi|}{M_{s}} \ll 1$ because $\left(\frac{\hat{h}}{\lambda}\right)$ is suppressed. Also, $\xi_{\text {eff }}$ is given by the expression:

$$
\xi_{e f f}=-\frac{Q_{m}}{4 \pi^{2}}\left\langle\partial_{T_{m}} K\right\rangle
$$

For $K=m_{p}^{2}\left(-n_{m} \log \left(T_{m}+\bar{T}_{m}\right)+\ldots\right)$ with $n_{m}=\mathcal{O}(1)$ occurring in string compactifications, one gets:

$$
\xi_{\text {eff }} \sim \frac{n_{m} Q_{m}}{8 \pi^{2}\left\langle\tau_{m}\right\rangle} m_{p}^{2} \lesssim M_{s}^{2}
$$

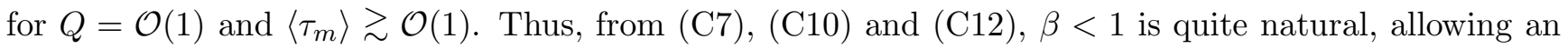
expansion of $|\psi|$ in powers of $\beta / 4$. This also implies that $|\psi| \lesssim M_{s}$ from (C7). Thus, we have checked that our solution is consistent with all requirements on the field space as in (C3).

\section{APPENDIX D: $D$-TERM CONTRIBUTION TO MASSES OF MODULI}

We saw in section IV and in the previous section that $U(1) D$-terms depend on the moduli. An effective Fayet-Iliopoulos (FI) parameter arises when these moduli are stabilized. In order to not destabilize the minima obtained from the $F$-term potential, one would like all the $D$-terms to vanish. This will give rise to additional constraints on the moduli in general. Two kinds of situations can arise. If the moduli appearing in the $D$-terms are not stabilized by other effects such as higher order corrections to the kähler potential, then the requirement of a vanishing $D$-term can stabilize the moduli if the vacuum expectation values (vevs) of charged matter fields are determined by other considerations. In the second situation, it could happen that the moduli are stabilized by other effects such as higher order corrections to the kähler potential. In this case, the vevs of charged matter fields could be determined in terms of the stabilized moduli. For the $U(1)_{B-L} D$-term studied in the previous section, we have assumed the second case. In both situations, one could look at fluctuations around the minima of the moduli (where the $D$-terms vanish) and compute their masses. It turns out that the $D$-term contribution to the masses of these moduli are generically much larger than $m_{3 / 2}$, as we argue below. This is also supported by arguments given in [59].

We study the $D$-term contribution to the potential around the minimum. For concreteness, we will study the $U(1)_{B-L} D$-term studied in the previous section and compute the mass of the modulus $T_{m}$. This is given by:

$$
\begin{aligned}
V_{D} & =\left\langle V_{D}\right\rangle+\delta V_{D} \\
& =\frac{m_{p}^{2} Q_{m}^{2} n_{m}^{2}}{\left(8 \pi^{2}\right)^{2}\left\langle\tau_{m}\right\rangle^{2}}\left(\delta \hat{T}_{m}\right)^{2}
\end{aligned}
$$

Here we have assumed that the $D$-term potential vanishes at the minimum and that $K=$ $m_{p}^{2}\left[-n_{m} \log \left(T_{m}+\bar{T}_{m}\right)+\ldots\right]$. Since the mass matrix of canonically normalized moduli $X_{i}$ is given by $\hat{m}_{i j}^{2}=K_{i j}^{-1} m_{i j}^{2}$ where $m_{i j}^{2}$ is the mass matrix of the un-normalized moduli, the mass of the canonically 
normalized modulus $T_{m}$ is given by:

$$
\hat{m}_{T_{m}} \approx \frac{n_{m}^{1 / 2} Q_{m} m_{p}}{8 \pi^{2}} \gtrsim 10^{-2} m_{p}
$$

for $n_{m}, Q_{m}=\mathcal{O}(1)$. Thus, the $D$-term contribution to the moduli masses are much larger than $m_{3 / 2}$.

[1] M. Fukugita and T. Yanagida, Phys. Lett. B 174, 45 (1986).

W. Buchmuller, R. D. Peccei and T. Yanagida, Ann. Rev. Nucl. Part. Sci. 55, 311 (2005) arXiv:hep-ph/0502169.

S. Davidson, E. Nardi and Y. Nir, arXiv:0802.2962 [hep-ph].

S. Davidson and A. Ibarra, Phys. Lett. B 535, 25 (2002) arXiv:hep-ph/0202239.

G. F. Giudice, A. Notari, M. Raidal, A. Riotto and A. Strumia, Nucl. Phys. B 685, 89 (2004) arXiv:hep-ph/0310123.

[2] G. D'Ambrosio, G. F. Giudice and M. Raidal, Phys. Lett. B 575, 75 (2003) arXiv:hep-ph/0308031.

Y. Grossman, T. Kashti, Y. Nir and E. Roulet, JHEP 0411, 080 (2004) arXiv:hep-ph/0407063.

Y. Grossman, R. Kitano and H. Murayama, JHEP 0506, 058 (2005) arXiv:hep-ph/0504160.

[3] I. Affleck and M. Dine, Nucl. Phys. B 249, 361 (1985).

M. Dine, L. Randall and S. D. Thomas, Nucl. Phys. B 458, 291 (1996) arXiv:hep-ph/9507453.

[4] K. Hamaguchi, H. Murayama and T. Yanagida, Phys. Rev. D 65, 043512 (2002) arXiv:hep-ph/0109030.

H. Murayama and T. Yanagida, Phys. Lett. B 322, 349 (1994) arXiv:hep-ph/9310297.

[5] A. G. Cohen, D. B. Kaplan and A. E. Nelson, Ann. Rev. Nucl. Part. Sci. 43, 27 (1993) arXiv:hep-ph/9302210].

V. A. Rubakov and M. E. Shaposhnikov, Usp. Fiz. Nauk 166, 493 (1996) [Phys. Usp. 39, 461 (1996)] arXiv:hep-ph/9603208.

A. Riotto and M. Trodden, Ann. Rev. Nucl. Part. Sci. 49, 35 (1999) arXiv:hep-ph/9901362.

M. S. Carena, J. M. Moreno, M. Quiros, M. Seco and C. E. M. Wagner, Nucl. Phys. B 599, 158 (2001) arXiv:hep-ph/0011055.

P. Huet and A. E. Nelson, Phys. Rev. D 53, 4578 (1996) arXiv:hep-ph/9506477.

[6] T. Banks, D. B. Kaplan and A. E. Nelson, Phys. Rev. D 49, 779 (1994) arXiv:hep-ph/9308292.

B. de Carlos, J. A. Casas, F. Quevedo and E. Roulet, Phys. Lett. B 318, 447 (1993) arXiv:hep-ph/9308325.

G. D. Coughlan, W. Fischler, E. W. Kolb, S. Raby and G. G. Ross, Phys. Lett. B 131, 59 (1983).

T. Banks, M. Berkooz and P. J. Steinhardt, Phys. Rev. D 52, 705 (1995) arXiv:hep-th/9501053.

T. Banks, M. Berkooz, S. H. Shenker, G. W. Moore and P. J. Steinhardt, Phys. Rev. D 52, 3548 (1995) arXiv:hep-th/9503114.

A. D. Linde, Phys. Rev. D 53, 4129 (1996) arXiv:hep-th/9601083.

T. Barreiro, B. de Carlos and N. J. Nunes, Phys. Lett. B 497, 136 (2001) arXiv:hep-ph/0010102.

[7] M. Fujii, K. Hamaguchi and T. Yanagida, Phys. Rev. D 65, 043511 (2002) arXiv:hep-ph/0109154.

M. Fujii, K. Hamaguchi and T. Yanagida, Phys. Rev. D 64, 123526 (2001) arXiv:hep-ph/0104186.

[8] M. Fujii, K. Hamaguchi and T. Yanagida, Phys. Rev. D 63, 123513 (2001) arXiv:hep-ph/0102187.

[9] K. Dasgupta, G. Rajesh and S. Sethi, JHEP 9908, 023 (1999) arXiv:hep-th/9908088.

S. B. Giddings, S. Kachru and J. Polchinski, Phys. Rev. D 66, 106006 (2002) arXiv:hep-th/0105097.

S. Kachru, R. Kallosh, A. Linde and S. P. Trivedi, Phys. Rev. D 68, 046005 (2003) arXiv:hep-th/0301240.

F. Denef, M. R. Douglas, B. Florea, A. Grassi and S. Kachru, Adv. Theor. Math. Phys. 9, 861 (2005) arXiv:hep-th/0503124.

[10] V. Balasubramanian, P. Berglund, J. P. Conlon and F. Quevedo, JHEP 0503, 007 (2005) arXiv:hep-th/0502058. 
[11] B. S. Acharya, arXiv:hep-th/0212294.

[12] B. Acharya, K. Bobkov, G. Kane, P. Kumar and D. Vaman, Phys. Rev. Lett. 97, 191601 (2006) arXiv:hep-th/0606262.

B. S. Acharya, K. Bobkov, G. L. Kane, P. Kumar and J. Shao, Phys. Rev. D 76, 126010 (2007) arXiv:hep-th/0701034.

[13] K. Choi, A. Falkowski, H. P. Nilles, M. Olechowski and S. Pokorski, JHEP 0411, 076 (2004) arXiv:hep-th/0411066.

K. Choi, A. Falkowski, H. P. Nilles and M. Olechowski, Nucl. Phys. B 718, 113 (2005) arXiv:hep-th/0503216].

K. Choi, K. S. Jeong and K. i. Okumura, JHEP 0509, 039 (2005) arXiv:hep-ph/0504037.

O. Loaiza-Brito, J. Martin, H. P. Nilles and M. Ratz, AIP Conf. Proc. 805, 198 (2006) arXiv:hep-th/0509158.

M. Endo, M. Yamaguchi and K. Yoshioka, Phys. Rev. D 72, 015004 (2005) arXiv:hep-ph/0504036.

S. Kachru, J. McGreevy and P. Svrcek, JHEP 0604, 023 (2006) arXiv:hep-th/0601111.

[14] M. Haack, D. Krefl, D. Lust, A. Van Proeyen and M. Zagermann, JHEP 0701 (2007) 078 arXiv:hep-th/0609211.

[15] R. Blumenhagen, S. Moster and E. Plauschinn, JHEP 0801, 058 (2008) [arXiv:0711.3389 [hep-th]].

[16] M. Cicoli, J. P. Conlon and F. Quevedo, JHEP 0810, 105 (2008) [arXiv:0805.1029 [hep-th]].

[17] B. Greene, S. Judes, J. Levin, S. Watson and A. Weltman, JHEP 0707, 060 (2007) arXiv:hep-th/0702220.

L. Kofman, A. Linde, X. Liu, A. Maloney, L. McAllister and E. Silverstein, JHEP 0405, 030 (2004) arXiv:hep-th/0403001.

S. Watson, Phys. Rev. D 70, 066005 (2004) arXiv:hep-th/0404177.

[18] J. P. Conlon and F. Quevedo, JHEP 0606, 029 (2006) arXiv:hep-th/0605141.

[19] O. Lebedev, H. P. Nilles and M. Ratz, Phys. Lett. B 636, 126 (2006) arXiv:hep-th/0603047.

[20] D. E. Diaconescu, B. Florea, S. Kachru and P. Svrcek, JHEP 0602, 020 (2006) arXiv:hep-th/0512170].

E. Floratos and C. Kokorelis, arXiv:hep-th/0607217.

[21] T. Kawano, H. Ooguri and Y. Ookouchi, Phys. Lett. B 652, 40 (2007) arXiv:0704.1085] [hep-th]].

J. J. Heckman, C. Vafa, H. Verlinde and M. Wijnholt, JHEP 0806, 016 (2008) [arXiv:0711.0387 [hep-ph]].

C. Beasley, J. J. Heckman and C. Vafa, JHEP 0901, 059 (2009) [arXiv:0806.0102 [hep-th]].

J. J. Heckman, J. Marsano, N. Saulina, S. Schafer-Nameki and C. Vafa, arXiv:0808.1286 [hep-th].

J. Marsano, N. Saulina and S. Schafer-Nameki, arXiv:0808.1571 [hep-th].

[22] J. P. Conlon and F. Quevedo, JCAP 0708, 019 (2007) arXiv:0705.3460 [hep-ph]].

[23] B. S. Acharya, P. Kumar, K. Bobkov, G. Kane, J. Shao and S. Watson, JHEP 0806, 064 (2008) arXiv:0804.0863 [hep-ph]].

[24] M. Dine, L. Randall and S. D. Thomas, Nucl. Phys. B 458, 291 (1996) arXiv:hep-ph/9507453.

[25] R. Blumenhagen, M. Cvetic and T. Weigand, Nucl. Phys. B 771, 113 (2007) arXiv:hep-th/0609191.

L. E. Ibanez and A. M. Uranga, JHEP 0703, 052 (2007) arXiv:hep-th/0609213.

M. Cvetic, R. Richter and T. Weigand, Phys. Rev. D 76, 086002 (2007) arXiv:hep-th/0703028.

R. Blumenhagen, M. Cvetic, D. Lust, R. Richter and T. Weigand, Phys. Rev. Lett. 100, 061602 (2008) arXiv:0707.1871 [hep-th]].

[26] K. Enqvist and A. Mazumdar, Phys. Rept. 380, 99 (2003) arXiv:hep-ph/0209244.

[27] R. Brustein and P. J. Steinhardt, Phys. Lett. B 302, 196 (1993) arXiv:hep-th/9212049.

[28] N. Kaloper and K. A. Olive, Astropart. Phys. 1, 185 (1993).

G. Huey, P. J. Steinhardt, B. A. Ovrut and D. Waldram, Phys. Lett. B 476, 379 (2000) arXiv:hep-th/0001112.

T. Battefeld and S. Watson, Rev. Mod. Phys. 78, 435 (2006) arXiv:hep-th/0510022.

[29] J. P. Conlon, R. Kallosh, A. Linde and F. Quevedo, arXiv:0806.0809 [hep-th].

[30] T. Asaka, M. Fujii, K. Hamaguchi and T. Yanagida, Phys. Rev. D 62, 123514 (2000) arXiv:hep-ph/0008041].

[31] A. Kusenko and M. E. Phys. Lett. B 418, 46 (1998) arXiv:hep-ph/9709492.

[32] V. A. Kuzmin, V. A. Rubakov and M. E. Shaposhnikov, Phys. Lett. B 155, 36 (1985). 
[33] D. J. H. Chung, B. Garbrecht and S. Tulin, arXiv:0807.2283 [hep-ph].

[34] S. Nakamura and M. Yamaguchi, Phys. Lett. B 638, 389 (2006) arXiv:hep-ph/0602081.

M. Endo, K. Hamaguchi and F. Takahashi, Phys. Rev. Lett. 96, 211301 (2006) arXiv:hep-ph/0602061.

M. Endo, K. Hamaguchi and F. Takahashi, Phys. Rev. D 74, 023531 (2006) arXiv:hep-ph/0605091.

[35] M. Ibe and R. Kitano, JHEP 0708, 016 (2007) arXiv:0705.3686 [hep-ph]].

T. Gherghetta, G. F. Giudice and A. Riotto, Phys. Lett. B 446, 28 (1999) arXiv:hep-ph/9808401.

[36] M. Endo and F. Takahashi, Phys. Rev. D 74, 063502 (2006) arXiv:hep-ph/0606075].

M. Nagai and K. Nakayama, Phys. Rev. D 76, 123501 (2007) arXiv:0709.3918 [hep-ph]].

[37] K. Choi, K. S. Jeong and K. I. Okumura, JHEP 0807, 047 (2008) arXiv:0804.4283 [hep-ph]].

[38] L. E. Ibanez, A. N. Schellekens and A. M. Uranga, JHEP 0706, 011 (2007) arXiv:0704.1079 [hep-th]].

[39] L. E. Ibanez and A. M. Uranga, JHEP 0802, 103 (2008) arXiv:0711.1316 [hep-th]].

D. Forcella, I. Garcia-Etxebarria and A. Uranga, arXiv:0806.2291 [hep-th].

[40] S. Antusch, L. E. Ibanez and T. Macri, JHEP 0709, 087 (2007) arXiv:0706.2132 [hep-ph]].

[41] S. Antusch and S. F. King, New J. Phys. 6, 110 (2004) arXiv:hep-ph/0405272.

[42] A. Brignole, L. E. Ibanez and C. Munoz, arXiv:hep-ph/9707209.

[43] J. P. Conlon, JHEP 0803, 025 (2008) arXiv:0710.0873 [hep-th]].

[44] J. P. Conlon, D. Cremades and F. Quevedo, JHEP 0701, 022 (2007) arXiv:hep-th/0609180.

[45] D. Cremades, L. E. Ibanez and F. Marchesano, JHEP 0405, 079 (2004) arXiv:hep-th/0404229.

M. Cvetic and I. Papadimitriou, Phys. Rev. D 68, 046001 (2003) [Erratum-ibid. D 70, 029903 (2004)] arXiv:hep-th/0303083.

[46] N. Arkani-Hamed, L. J. Hall, H. Murayama, D. Tucker-Smith and N. Weiner, Phys. Rev. D 64, 115011 (2001) arXiv:hep-ph/0006312.

Z. Thomas, D. Tucker-Smith and N. Weiner, Phys. Rev. D 77, 115015 (2008) [arXiv:0712.4146 [hep-ph]].

[47] R. Bernabei et al. [DAMA Collaboration], arXiv:0804.2741 [astro-ph].

[48] D. Tucker-Smith and N. Weiner, Phys. Rev. D 72, 063509 (2005) arXiv:hep-ph/0402065.

S. Chang, G. D. Kribs, D. Tucker-Smith and N. Weiner, arXiv:0807.2250 [hep-ph].

[49] M. L. Graesser, arXiv:0705.2190 [hep-ph].

[50] S. G. Frederiksen, N. Johnson, G. L. Kane and J. Reid, Phys. Rev. D 50, 4244 (1994).

D. Phalen, B. Thomas and J. D. Wells, Phys. Rev. D 75, 117702 (2007) arXiv:hep-ph/0612219.

S. Gopalakrishna, S. Jung and J. D. Wells, arXiv:0801.3456 [hep-ph].

[51] M. Fujii, K. Hamaguchi and T. Yanagida, Phys. Lett. B 538, 107 (2002) arXiv:hep-ph/0203189.

[52] B. Acharya and E. Witten, arXiv:hep-th/0109152.

[53] M. Atiyah and E. Witten, Adv. Theor. Math. Phys. 6, 1 (2003) arXiv:hep-th/0107177.

M. Cvetic, G. Shiu and A. M. Uranga, arXiv:hep-th/0111179.

[54] R. Kitano, H. Murayama and M. Ratz, arXiv:0807.4313 [hep-ph].

S. D. Thomas, Phys. Lett. B 356, 256 (1995) arXiv:hep-ph/9506274.

[55] B. Acharya, R. Howl, G. Kane, S. King and P. Kumar, Work in Progress.

[56] H. Murayama and Y. Nomura, Phys. Rev. D 75, 095011 (2007) arXiv:hep-ph/0701231.

[57] R. Kitano, Phys. Lett. B 641, 203 (2006) arXiv:hep-ph/0607090.

[58] R. Allahverdi and M. Drees, Phys. Rev. D 69, 103522 (2004) arXiv:hep-ph/0401054.

[59] G. Villadoro and F. Zwirner, JHEP 0603, 087 (2006) arXiv:hep-th/0602120. 